\title{
Single-Cell Analysis of Tumor-Specific Neutrophils During Gastric Cancer Progression Inspired a Hippo Targeted Antitumor Therapy
}

Shi Jiao ( $\nabla_{\text {jiaoshi@sibcb.ac.cn ) }}$

Fudan University

Pingping Nie

SIBCB

\section{Weihong Zhang}

Tongji University

\section{Yang Meng}

Shanghai Eastern Hepatobiliary Surgery Hospital

\section{Fenghua Guo}

Hua'shan Hospital

\section{Hui Zhang}

State Key Laboratory of Cell Biology, CAS Center for Excellence in Molecular Cell Science, Institute of Biochemistry and Cell Biology, Shanghai Institutes for Biological Sciences, CAS

\section{Zhenzhu Tong}

SIBCB

\section{Meng Wang}

Fudan University

\section{Fan Chen}

SIBCB

\section{Liwei An}

Tongji University

\section{Yi Han}

Tongji University

\section{Wenjia Wang}

Institute of Biochemistry and Cell Biology, Shanghai Institutes for Biological Sciences, Chinese Academy of Sciences

\section{Lixin Wei}

\section{Eastern Hepatobiliary Surgery Hospital}

\section{Zhaocai Zhou}

State Key Laboratory of Cell Biology, CAS Center for Excellence in Molecular Cell Science, Shanghai Institute of Biochemistry and Cell Biology, Chinese Academy of Sciences, University of Chinese Acade 
https://orcid.org/0000-0002-5441-3922

\section{Article}

Keywords: neutrophils, gastric cancer, tumorigenesis

Posted Date: May 27th, 2021

DOl: https://doi.org/10.21203/rs.3.rs-533205/v1

License: (c) (i) This work is licensed under a Creative Commons Attribution 4.0 International License. Read Full License 


\section{Single-Cell Analysis of Tumor-Specific Neutrophils During Gastric Cancer Progression Inspired a Hippo Targeted Antitumor Therapy}

Pingping Nie ${ }^{1,2,7}$, Weihong Zhang ${ }^{3,4,7}$, Yan Meng ${ }^{5,7}$, Fenghua Guo ${ }^{6}$, Hui Zhang ${ }^{1,2}$, Zhenzhu

Tong $^{1}$, Meng Wang ${ }^{2}$, Fan Chen ${ }^{1}$, Liwei $\mathrm{An}^{3}, \mathrm{Yi} \mathrm{Han}^{3}$, Wenjia Wang ${ }^{2}$, Linxin Wei ${ }^{{ }^{*}}$, Zhaocai Zhou $^{2^{*}}$, Shi Jiao ${ }^{2^{*}}$

${ }^{1}$ CAS Center for Excellence in Molecular Cell Science, Institute of Biochemistry and Cell Biology, Shanghai Institutes for Biological Sciences, Chinese Academy of Sciences, University of Chinese Academy of Sciences, Shanghai 200031

${ }^{2}$ State Key Laboratory of Genetic Engineering, School of Life Sciences, Zhongshan Hospital, Fudan University, Shanghai 200438, China

${ }^{3}$ Tongji University Cancer Center, Department of Medical Ultrasound, Shanghai Tenth People's Hospital, Ultrasound Research and Education Institute, Tongji University School of Medicine, Shanghai, 200072, China

${ }^{4}$ Postdoctoral Station of Clinical Medicine, Shanghai Tenth People's Hospital, Tongji University School of Medicine, Shanghai, 200072, China

${ }^{5}$ Tumor Immunology and Gene Therapy Center, Shanghai Eastern Hepatobiliary Surgery Hospital, Shanghai 200438, China

${ }^{6}$ Department of General Surgery, Hua'shan Hospital, Fudan University Shanghai Medical College, Shanghai, 200040, China

${ }^{7}$ These authors contribute equally to this work.

${ }^{*}$ Correspondence:

Dr. Shi Jiao (jiaoshi@sibcb.ac.cn), Dr. Zhaocai Zhou (zczhou@sibcb.ac.cn) and Dr. Linxin Wei (weilixin_smmu@163.com),. Address, No. 2005 Song-Hu Road, Shanghai 200438, China. 


\section{ABSTRACT}

Neutrophils are important for tumor immunosurveillance; yet their roles in tumorigenesis remain debating due to incomplete understanding of their heterogeneity and functional plasticity. Here we resolve at single-cell resolution the heterogeneity of neutrophils in tumorbearing mice and patients with gastric cancer. Our results define $C X C R 22^{\text {neg }} C D 44^{\text {neg }}$ neutrophils as a tumor-specific (tsNeu) population with activated ( $\mathrm{CD} 14^{\text {high }}$ ) but atavistic phenotypes $\left(\mathrm{CD} 53^{\text {high }} \mathrm{CD} 63^{\text {high }}\right)$. Human and mouse tsNeus can be further classified into four subsets according to their expression of CD54 and CD101. Importantly, the spatiotemporal evolution and anti-tumor function of tsNeu requires the Hippo pathway transcriptional co-activator YAP/TAZ. Deficiency of YAP/TAZ impairs the generation of effective tsNeus and therefore accelerates tumor progression. Remarkably, treatment with the Hippo antagonist phosphatidic acid (PA) turns blood neutrophils into tsNeus with enhanced abilities of direct killing tumor cells, as well as inducing adaptive anti-tumor immunity. Collectively, our work not only identified molecular makers for tsNeus, but also revealed key transcriptional programs for their fate determination and function; and further offers a strategy of targeting Hippo-YAP/TAZ for neutrophil therapy against gastric cancer. 


\section{INTRODUCTION}

Neutrophils serve as the first line of host defense against pathogen infection and tissue injury. In response to inflammatory stimuli, neutrophils migrate from the peripheral blood to infected or injured tissues ${ }^{1}$. Neutrophils are also found in the tumor microenvironment and associated with tumor progression in a variety of cancers ${ }^{2}$. However, the specific role of neutrophils in cancer initiation and progression has long been under controversy: tumor associated neutrophils appear to exert dual functions with either anti-tumor or pro-tumor effect.

The anti-tumor effects of neutrophils include not only cytotoxicity and innate immunity, but also the induction of adaptive immunity ${ }^{3,4}$. For example, neutrophils directly kill tumor cells via ROS production and $\mathrm{H}_{2} \mathrm{O}_{2}$-mediated cytotoxicity ${ }^{5,6,7}$. As a key player of innate immunity, neutrophils can restrict bacterial infiltration to inhibit intestinal microbiota driven tumor-promoting inflammation and colorectal carcinogenesis ${ }^{8,9}$. Moreover, tumor-associated neutrophils acquire an activated phenotype with characteristics of antigen-presenting cells to stimulate conventional $\mathrm{CD} 4^{+}$and $\mathrm{CD} 8^{+} \mathrm{T}$ cell proliferation and production of effector cytokines 10, 11. In addition, neutrophils may cooperate with macrophages to promote the activation of CD4 CD8 unconventional $\alpha \beta$ T cells and IFN production ${ }^{12,13}$, as well as to suppress interleukin-17A (IL-17A) production by tumor-promoting $\gamma \delta$ T cells ${ }^{13}$.

Neutrophils can also exert pro-tumor effects including angiogenesis, extracellular matrix remodeling, distant metastasis and immune-suppression. For instance, neutrophils promote angiogenesis by the production of pro-angiogenic factors ${ }^{14,15}$. Neutrophil extracellular trap (NET)-associated proteases such as elastase MMP9 cleave the extracellular matrix protein laminin and induce the proliferation of dormant cancer cells ${ }^{16}$. Neutrophils can escort circulating tumor cells (CTCS) and drive cell cycle progression within the bloodstream to enhance the metastatic potential of CTCS ${ }^{17}$. Moreover, DNA component of NETs acts as a chemotactic factor to attract cancer cell for distant metastasis ${ }^{18,19}$. Neutrophils not only inhibit natural killer cytotoxicity to tumor cells ${ }^{20}$, but also suppress effector T cell-mediated antitumor response by production of ROS, NO, prostaglandin E2 and upregulation of programmed cell death 1 ligand 1 (PDL1) 21, 22, 23.

The paradoxical phenotypes of neutrophils largely arise from the enormous spatiotemporal heterogeneity concerning their maturation, activation, half-life, aging status, tissue distribution and effector function diversity ${ }^{24,25}$. Neutrophils are derived from common myeloid progenitors (CMPs) through granulocyte monocytes progenitors (GMPs) ${ }^{26}$. During differentiation and maturation, neutrophils undergo a series of morphological changes: myeloblast, promyelocyte, myelocytes, metamyelocytes and band cells ${ }^{27}$. Recent single cell RNA sequencing (scRNA-Seq) analysis defined eight neutrophil subpopulations from bone marrow, peripheral blood and spleen, with distinct maturing bone marrow neutrophil subsets giving rise to different mature peripheral neutrophil subsets ${ }^{28}$. Moreover, a very early unipotent neutrophil progenitor termed "eNeP" has been defined as $\mathrm{Lin}^{-} \mathrm{CD} 66 \mathrm{~b}^{+} \mathrm{c}-\mathrm{Kit}^{+} \mathrm{CD} 71^{+}$ 
in human bone marrow, in parallel with GMPs of mice ${ }^{29}$. Meanwhile, scRNA-Seq analysis identified $\mathrm{Ly} \mathrm{C}^{+} \mathrm{CD} 115^{-} \mathrm{CD} 81^{+} \mathrm{CD} 11 \mathrm{~b} \mathrm{C}^{-} \mathrm{CD} 106^{-} \mathrm{GMP}$ as an early committed neutrophil progenitor $(\text { proNeu1 })^{30}$. ProNeu1 sequentially differentiates into proNeu2 and then neutrophil precursor (preNeu), which are committed to non-proliferative immature and then mature neutrophils ${ }^{31}$.

Differentiation and maturation of neutrophils are orchestrated by various transcription factors involved at different stages of development ${ }^{28}$. For example, C/EBP $\alpha$ which is highly expressed in GMPs, not only plays an import role in the initiation of granulopoiesis, but also is required for the differentiation of proNeu1 to proNeu2 and then preNeu ${ }^{31}$. Growth factor independent 1 (GFI-1), which is highly expressed in proNeu2 and preNeu, is essential for early neutrophil differentiation ${ }^{32}$. PU.1 and C/EBPS are involved in terminal granulopoiesis and expressed in bone marrow mature neutrophils, as wells as in peripheral blood circulating neutrophils. Various granule proteins are differentially synthesized and stored: primary granules such as Mpo, Elane and Ctsg are highly expressed in GMPs and proNeus; secondary granules such as Ltf, Camp and Lcn2 are expressed within preNeus and bone marrow immature neutrophils; tertiary granules such as $M m p 8, M m p 9$ and Itgam are formed within bone marrow mature and peripheral neutrophils.

In contrast to the well-studied neutrophil development under physiological condition or infection, the maturation and activation of neutrophils are poorly understood in the case of tumorigenesis. For example, neutrophil heterogeneity remains to be fully characterized in the tumor microenvironment; the evolution routes during tumor progression and the transcriptional program for their fate decision are largely unknown. Therefore, therapeutic targeting of neutrophils is underdeveloped. Here, we performed a comprehensive study of neutrophils associated with gastric cancer (GC) via combining scRNA-Seq with mice tumor model, and clinical GC sample, which defined tumor-specific neutrophils (tsNeu) as a CXCR2 ${ }^{\text {neg }} C D 44^{\text {neg }}$ population with four subsets marked by CD54 and CD101. We further demonstrated that YAP/TAZ are required for the generation and antitumor function of tsNeus. Based on these findings, we developed a Hippo-YAP-targeted neutrophil therapy to treat GC. 


\section{RESULTS}

\section{scRNA-Seq Analysis of Ly6G $^{+}$Neutrophils in Mice Bearing GC}

To characterize tissue-specific neutrophils during tumorigenesis, we isolated by fluorescenceactivated cell sorting (FACS) $\mathrm{CD}_{4} 5^{+} \mathrm{CD} 11 \mathrm{~b}^{+} \mathrm{Ly}_{6 \mathrm{G}}{ }^{+}$cells to exclude $\mathrm{Ly} 6 \mathrm{G}^{\text {neg }}$ neutrophiles (GMP and proNeu) from bone marrow (BM), spleen (SP), peripheral blood (PB) and gastric cancer (GC) tissue of mice bearing GC (Fig. 1a). After rigorous quality control, we obtained 22,069 high-quality cells with an average of 1,189 genes per cell profiled, with a total of 17,458 genes detected (Supplementary Fig. 1a-c, Supplementary Table 1). Using genome-wide correlations between cluster mean expression and previously defined transcriptional profiles of sorted neutrophil subsets ${ }^{30,33,34}$ and canonical markers (Supplementary Table S2), we determined nine major cell clusters (Fig. 1b upper). Note that both of the two clusters of $L y 6 c^{h i} I \operatorname{tg} b 3^{h i}$ preneutrophil (preNeu1/2) are mainly from bone marrow and spleen; while both the $\mathrm{Ngp}^{\text {hi }} \mathrm{Cxcr} 2^{10}$ immature neutrophil (immNeu) and the $\mathrm{C} x \mathrm{Cr} 2^{\text {hi }} \mathrm{Mmp} 8^{\text {hi }}$ mature neutrophil ( $\mathrm{mNeu}$ ) are mainly from spleen and peripheral blood (Fig. 1B, Supplementary Fig. 1d). These findings are consistent with previous studies ${ }^{30,33,34}$ and verify the robustness of the scRNA-Seq analysis in this work.

In contrast to the overlapping of cell clusters from bone marrow, spleen and peripheral blood, the transcriptomic map of most tumor-specific neutrophils (tsNeus) appears to be well separated from that of neutrophils in other tissues (Fig. 1b lower). Dimensional reduction with uniform manifold approximation and projection (UMAP) further revealed four distinct clusters of tsNeus, which we termed tsNeu1-4 (Fig.1b, Supplementary Fig. 1d). Moreover, we found the clusters of tsNeu1/2 were $\mathrm{Cd} 44^{10} / \mathrm{cam} 1{ }^{10} \mathrm{Gdf15} 5^{10}$, whereas the clusters of tsNeu3/4 were Cd $44^{10} / \mathrm{cam} 1^{\text {hi }} \mathrm{Gdf} 15^{\text {hi }}$ (Fig. 1c, Supplementary Fig. 1d, Supplementary Table 2). In addition, we also noticed a highly heterogeneous cell cluster consisting of neutrophils from all four examined tissues, which we termed silent neutrophils (s $\mathrm{Neu}$ ) expressing early markers for neutrophil development such as Elane and Prtn3 (Fig. 1b, Supplementary Fig. 1d, Supplementary Table 2).

To resolve the lineage of ts Neu relative to neutrophils in other tissues, we performed Monocle pseudo-time analysis ${ }^{35}$. Consistent with the above UMAP analysis, tsNeu was found to be closely associated with neutrophil subsets in peripheral blood (immNeu and $\mathrm{mNeu}$ ) but more remotely associated with those in bone marrow and spleen (preNeu1/2) (Fig. 1d). Furthermore, we performed principal-component analysis (PCA) to compare gene expression profiles of all the above identified neutrophil subsets. Consistent with previous studies ${ }^{33,34}$, the subsets preNeu1, preNeu2, immNeu and $\mathrm{mNeu}$ displayed distinct gene signatures (Fig. 1e). Notably, tsNeu1 and tsNeu2 displayed similar gene expression profiles with immNeu and mNeu, respectively (Fig. 1e). Moreover, tsNeu3/4 showed significant transcriptomic differences compared to tsNeu1/2 (Fig. 1e). Interestingly, although sNeu were Ly6G', these neutrophils showed a similar transcriptional profile with that of GMP, which is well identified 
as a subset of Ly6G- neutrophils (Fig. 1e).

Neutrophil differentiation occurred on a tightly organized trajectory, starting from GMP cells in bone marrow and spleen to $\mathrm{mNeu}$ in spleen and peripheral blood. A cluster of imm Neu cells followed preNeu expansion and expressed secondary granule genes such as $\mathrm{Ngp}$ (Supplementary Fig. 1d), and this cluster migrated from bone marrow to the peripheral blood. Neutrophil differentiation in bone marrow tended to end with mNeu highly expressing $\mathrm{Mmp} 8$ (Supplementary Fig. 1d). RNA velocity analysis, a method inferring precursor-progeny cell dynamics revealed that preNeu subsets exhibited a strong directional flow towards immNeu and $\mathrm{mNeu}$ (Fig. 1f), indicating that neutrophil maturation (from preNeu to $\mathrm{mNeu}$ ) follows a single main branch without significant division. We also observed a clear directional flow from immNeu and mNeu enriched in peripheral blood to tsNeu enriched in tumor tissue (Figure 1F). Additionally, we observed that $7.3 \%$ (93 of 1,265$)$ sNeu had the potential to transit to tsNeu3/4 (Fig. 1f), suggesting that tsNeu3/4 may originate from both tsNeu1/2 and sNeu.

Neutrophil contains an assembly of granules destined for regulated secretion, each granule type with distinct constituents formed before terminal differentiation ${ }^{36}$. The earliest granules are designated primary (azurophil), followed in time by secondary (specific), and tertiary (gelatinase/secretory) granules ${ }^{37}$. To gain insights into the functional processes of Neutrophil that occur during tumor infiltration, we further analyzed the expression of various granule genes in tsNeus, the major population of neutrophils in the tumor tissue. tsNeus showed a highly coordinated expression of tertiary granules at levels comparable to that of mNeu (Fig. 1g). We also measured the levels of neutrophil maturation, aging and apoptosis based on their expression of related genes (Supplementary Table 3). Overall, the maturation levels of tsNeu are similar to those of immNeu and mNeu (Fig. 1g). Notably, tsNeu3/4 showed the highest scores of aging and apoptosis among all tumor-specific neutrophils, indicating a terminal bound identity (Fig. 1g, Supplementary Fig. 1e). tsNeu4 cluster showed the highest expression of key genes involved in reactive oxygen species (ROS) production, phagocytosis and chemotaxis (Fig. 1h).

Taken together, these results indicate that tsNeus represent a unique neutrophil population terminal to the trajectory along tumorigenesis; and that tsNeus are activated but exhibit an atavistic transcriptomic signature.

\section{Identification and Isolation of tsNeus by CD44 and CXCR2}

Our findings of tsNeu at the level of transcriptomic signatures promoted us to further characterize at the protein level the cell identity and state of this population. From the flow cytometric analysis, we observed preNeus $\left(\mathrm{CD} 11 \mathrm{~b}^{+} \mathrm{Gr} 1^{+} \mathrm{CXCR} 4^{+} \mathrm{c}-\mathrm{Kit}^{+}\right)$in tumor-bearing mice with a gradual upregulation of c-Kit and a subsequent bifurcation of c-Kit neutrophils into CD101 ${ }^{-}$and $\mathrm{CD} 101^{+}$cells, representing the previously characterized subsets of immNeu and $\mathrm{mNeu}$, respectively (Supplementary Fig. $2 \mathrm{a})^{33}$. Given the common origin of Ly6G+ly6C- 
neutrophils, however, these surface markers could not isolate tsNeus from other types of neutrophils (Supplementary Fig. 2a, b), challenging further study of distinct neutrophil subsets during tumorigenesis. To solve this issue, we systematically evaluated the expression levels of a group of cell surface markers, including neutrophil lineage-specific (c-kit, CD53, CD63, CD101, CXCR2, CXCR4, Ly6G, Ly6C), activation (CD14, CD54), rolling (CD44, CD62L), trans-endothelial migration (CD11b) markers $1,38,39$.

Consistent with our scRNA-Seq analysis indicating an activated but atavistic phenotype, tumor-infiltrated neutrophils showed high expression of Ly6G, CD53, CD54 (ICAM1), CD14, CD63, PDL1 and c-Kit, but low expression of Ly6C, CD62, CD44 and CXCR2 (Supplementary Fig. $2 \mathrm{~b}, \mathrm{c})$. We next performed a mini flow cytometric screening by gating different surface markers. Surprisingly, we observed a clear separation of tumor-infiltrated neutrophils from all other Ly6G'ly6C- neutrophils when sorted by CD44 and CXCR2 (Fig. 2a). Most tumor-infiltrated neutrophils (93.2\%) were CD44-CXCR2', and the majority (97.9\%) of CD44 CXCR2' neutrophils were tumor-infiltrated ones (Fig. 2a, Supplementary Fig. 2d). Moreover, most neutrophils in the peripheral blood were $\mathrm{CD} 44^{+} \mathrm{CXCR2}{ }^{+}$, while neutrophils in bone marrow and spleen consist of both $C D 44^{+} C X C R 22^{-}$and $C D 44^{+} C X C R 2^{+}$subsets (Fig. 2a). Together, these findings indicate that CD44 and CXCR2 can serve as specific surface markers to isolate tumor-infiltrated neutrophils

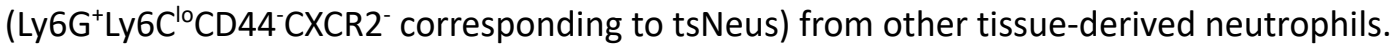

\section{Identification of Four tsNeu Subsets by CD54 and CD101}

To further dissect the heterogeneity of tsNeus, we chose CD54 (ICAM1) to segregate nonactivated and activated neutrophils, and CD101 to segregate immature and mature neutrophils. As such, we characterized four phenotypically distinct tsNeu subsets (CD101$\mathrm{CD}^{-}, \mathrm{CD} 101^{+} \mathrm{CD} 54^{-}, \mathrm{CD} 101^{-} \mathrm{CD} 54^{+}, \mathrm{CD} 101^{+} \mathrm{CD} 54^{+}$) (Fig. 2b), very likely corresponding to the four subsets defined by our scRNA-Seq analysis (tsNeu1 4)(Fig. 1). Morphological analysis with Wright-Giemsa Staining revealed a poly-segmented shape of the nucleus in the CD101CD54/tsNeu1 (immature-like shape, DN) and CD101 ${ }^{+} \mathrm{CD} 54^{-} / \mathrm{tsNeu2}$ (mature-like shape, SP1) subsets, with progressively increased cell area in the $\mathrm{CD} 101^{-} \mathrm{CD} 54^{+} / \mathrm{tsNeu} 3$ (SP2) and $\mathrm{CD} 101^{+} \mathrm{CD} 54^{+} /$tsNeu4 (DP) subsets (Fig. 2c). We then used LDH release assay to determine tsNeu-induced cytotoxicity towards tumor cell. As expected, treatment of MFC, a murine gastric cancer cell line with the sorted CD101 ${ }^{-}$D 54 $4^{-} /$tsNeu1 for $48 \mathrm{~h}$ induced an $\sim 10 \%$ increase of MFC cell death (Fig. 2d). Notably, CD54 $4^{+}$tsNeu showed stronger antitumor activity when compared with CD54tsNeu (Fig. 2d), findings consistent with the results of our scRNA-Seq analysis.

To determine the differentiation potential and route of the tsNeu subsets during tumorigenesis, we adoptively transferred the tsNeu subsets sorted from CD45.2 mice into CD45.1 mice. The results showed that CD54 ${ }^{-} \mathrm{tsNeu}$ (tsNeu1/2) gave rise to both CD54 tsNeus and $\mathrm{CD} 54^{+}$tsNeus after $48 \mathrm{~h}$; whereas $\mathrm{CD} 54^{+}$tsNeu (tsNeu3/4) only gave rise to $\mathrm{CD} 54^{+}$tsNeus (Fig. 2e). Moreover, we observed more apoptosis for $\mathrm{CD}_{5} 4^{+} \mathrm{tsNeu}$ (tsNeu3/4) than $\mathrm{CD} 54^{-} \mathrm{tsNeu}$ 
(tsNeu1/2) as indicated by Annexin V/PI staining, further confirming the above-transcriptomic analyses (Fig. 2f). Together with the scRNA-Seq analysis, these results demonstrated the heterogeneity of tsNeus, and further revealed a lineage commitment program governing the one-way differentiation of tsNeu1/2 to various tsNeu subsets.

\section{Time-based Evolution Trajectory of tsNeus along Tumor Progression}

To investigate the dynamic evolution of tsNeus along tumor progression, we analyzed the transcriptomic network of neutrophils from mice bearing GC for 3 or 10 days. With the same sequencing depth of scRNA-Seq, we observed an increase in both gene number and total UMIs in neutrophils isolated from mice bearing tumors for 10 days when compared with those from mice bearing tumors for 3 days, indicating elevated transcriptional activity in neutrophils during tumorigenesis (Supplementary Table 1). We performed t-distributed stochastic neighbor embedding (t-SNE) analysis ${ }^{40}$ and confirmed that tumor-infiltrated neutrophils were a mixed population of preNeu, immNeu, mNeu, ts Neu, and sNeu (Fig. 3a). We observed a decreased percentage of tsNeu1/2 but increased percentage of tsNeu3/4 in mice bearing tumors for 10 days when compared with those for 3 days (Fig. 3a, b), indicating transition of tsNeu1/2 into tsNeu3/4 along tumor progression. Consistent with the results of the above scRNA-Seq analysis, these clusters possessed key signature genes like CD63, CD54 (ICAM1) and Gdf15, and their gene expression correlated with the presence of tsNeu1/2, tsNeu3 and tsNeu4, respectively (Fig. 3c).

Subsequently, we verified at protein levels the expression of various surface markers in tumor-infiltrated neutrophils. We observed a significantly decreased number of total tumorinfiltrated neutrophils ( $\mathrm{Ly}_{6 \mathrm{G}} \mathrm{Cd}^{+} \mathrm{d} 11 \mathrm{~b}^{+} \mathrm{Ly} 6 \mathrm{C}^{\mathrm{lo}}$ ) in mice bearing $\mathrm{GC}$ for 10 days when compared with that for 3 days (Fig. 3d), indicating a rapid loss of tumor-infiltrated neutrophils along the disease progression. In contrast, we found the ratio of $\mathrm{CD}^{-}{ }^{-} \mathrm{CXCR} 2^{-}$tsNeu to total tumorinfiltrated neutrophils significantly increased from $88 \%$ in mice bearing GC for 3 days to $~ 97 \%$ in mice bearing GC for 10 days (Fig. 3e). In addition, we observed no significant change in the CD115 ${ }^{+}$Ly $6 \mathrm{G}^{+}$cells during tumorigenesis, a previously reported population of MDSCs ${ }^{41,} 42$ (Supplementary Fig. 3a). In keeping with the scRNA-Seq results (Fig. 3a, b), our flowcytometry analysis also revealed a similar change of the tsNeu population along tumor progression: decreased number of $\mathrm{CD}_{5} 4^{-} \mathrm{tsNeu}$ (tsNeu1/2) but increased number of $\mathrm{CD}_{5} 4^{+} \mathrm{tsNeu}$ (tsNeu3/4) in mice bearing GC for 10 days than those for 3 days (Fig. 3f).

Notably, the expression of CCL5, CD14, CD53 and CD63 in CD54 tsNeu (tsNeu3/4) from mice bearing GC for 10 days were significantly higher than that for 3 days (Supplementary Fig. 3b, 3c). In addition, we used early neutrophil-lineage markers, including CD34 and c-Kit, to isolate sNeu from total tumor-infiltrated neutrophils $\left(\mathrm{Ly}_{6 \mathrm{G}} \mathrm{H}^{\mathrm{L}} \mathrm{L} 6 \mathrm{C}^{\mathrm{lo}}\right.$ ). As shown in Supplementary Fig. 3d, the proportion of sNeu $\left(\mathrm{Ly}_{6 \mathrm{G}}{ }^{+} \mathrm{CD} 34^{+} \mathrm{c}-\mathrm{Kit}^{+}\right)$was significantly reduced from $\sim 2.0 \%$ in mice bearing GC for 3 days to $0.27 \%$ in mice bearing GC for 10 days, findings consistent with our scRNA-Seq analysis. Taken together, these results revealed a stepwise and 
hierarchical trajectory for tsNeu evolution during tumorigenesis.

\section{YAP/TAZ Is Required for the Antitumor Function of tsNeu}

To gain insights into the global gene regulatory networks involved in the lineage commitment of tsNeus, we performed SCENIC (single-cell regulatory network inference and clustering) analysis ${ }^{43}$ to compare the regulon activities between tsNeu and other neutrophil subsets, which identified 20 neutrophil-specific networks (Fig. 4a). As anticipated, preNeus highly expressed Gfi1 and Cebpe regulons, which are necessary for the early neutrophil development ${ }^{32}$ (Fig. 4a). In contrast, tsNeus highly expressed 6 regulons, including Egr1, Fosb, Balf, Irf5, Atf3 and Jun, which are associated with the activation of neutrophil ${ }^{34}$ (Fig. 4a). Notably, clustering analysis revealed high activity of Tead regulon in tsNeus (Fig. 4A). Moreover, the pattern of Tead regulon was similar to that of Atf3 and Jun (Supplementary Fig. 4a). Further gene set enrichment analysis (GSEA) demonstrated a global transcriptome change in tsNeus (relative to preNeus, immNeus, mNeus), with a significant positive enrichment of target genes of the YAP/TAZ-TEAD complex (Fig. 4b). Consistently, immunoblotting analysis showed that tumor-infiltrated Neu have the highest protein levels of YAP and TAZ in the four tissue samples (Fig. 4c). Importantly, the increase of YAP and TAZ reached a peak after implantation with MFC for 10 day, and then decreased until 15 days (Fig. 4d). In addition, tsNeu1/2 subsets (DN, SP1) showed higher activity of YAP/TAZ-TEAD complex when compared to tsNeu3/4 subsets (SP2, DP) (Fig. 4e), findings consistent with the above observations that tsNeu1/2 had lower apoptosis than tsNeu3/4 (Supplementary Fig. 1e, Fig. 2f).

Next, we cross-bred Yap $1^{\text {flox/flox }}$ mice and Taz ${ }^{\text {floxfflox }}$ mice with $L y z M^{\text {cre }}$ mice (termed as Yap1;Taz ${ }^{\text {DKO})}$ to generate mice with neutrophils deficient of YAP and TAZ (Fig. 4f). We did not observe a significant change for the development and total number of neutrophils in the Yap1;Taz ${ }^{D K O}$ mice compared to those of wildtype control littermates (Supplementary Fig. 4b). In contrast, in a subsequent xenograft tumor model, we observed much larger tumors in Yap1;Taz ${ }^{D K O}$ mice than in control mice, even though same amount of MFC tumor cells were inoculated (Fig. $\mathbf{4 g}$ ). To rule out the possibility that such results were caused by the nonspecific effects of $L y z M^{C r e}$, we adoptively transferred wildtype or Yap1;Taz ${ }^{D K O}$ neutrophils into wiltype mice loaded with tumors (Fig. 4h). As expected, the tumor volume was decreased after 3 times of intratumoral injection of neutrophils derived from wildtype mice when compared to the mock group (Fig. 4i). However, such effect was not observed for the group of neutrophils derived from Yap1;Taz ${ }^{D K O}$ mice, indicating a defect of tumor-killing ability for these neutrophils (Fig. 4i). Together, these results clearly indicate that YAP/TAZ is required for the antitumor function of neutrophils.

\section{YAP/TAZ Is Essential for Lineage Commitment of tsNeu}

We then examined the regulatory effects of YAP/TAZ towards various subsets of neutrophils during tumorigenesis. In the late stage of tumor progression, we observed a significantly decreased ratio of total tumor-infiltrated neutrophils $\left(\mathrm{Cd} 11 \mathrm{~b}^{+} \mathrm{Ly}_{6 \mathrm{G}^{+}} \mathrm{Ly}_{6 \mathrm{C}} \mathrm{C}^{\mathrm{lo}}\right.$ ) (Fig. 5a, 
Supplementary Fig. 5a), but a significant increased ratio of tumor-infiltrated preNeus (c$\mathrm{kit}^{+} \mathrm{CXCR}^{+}$) in Yap1;Taz ${ }^{\text {DKO }}$ mice (Supplementary Fig. 5b). However, YAP/TAZ deficiency did not affect the ratio of GMPs, preNeu, immNeu and mNeu in the bone marrow of tumorbearing mice (Supplementary Fig. 5c). Notably, the ratio of CD44-CXCR2 tsNeu to total tumorinfiltrated neutrophils significantly decreased from $\sim 97 \%$ in control mice to $\sim 41 \%$ in Yap1;Taz ${ }^{\text {KKO }}$ mice after bearing tumors for 20 days (Fig. 5b). Meanwhile, we observed an increased number of $\mathrm{CD} 101^{+} \mathrm{CD} 54^{-} / \mathrm{tsNeu} 2$ but a decreased number of $\mathrm{CD} 101^{-} \mathrm{CD} 54^{+} / \mathrm{tsNeu} 3$ and $\mathrm{CD} 101^{+} \mathrm{CD} 54^{+} / \mathrm{tsNeu4}$ in Yap1;Taz ${ }^{D K O}$ mice compared to the wildtype control mice after bearing tumors for 10 days (Fig. 5c). Moreover, YAP/TAZ deficiency reduced the expression levels of activation markers CD14, but enhanced the levels of early neutrophil lineage markers CD63 and c-Kit (Fig. 5d).

To investigate Hippo signaling-mediated immune roadmap to tumor progression, we isolated and sequenced a total of $10,858 \mathrm{CD} 45^{+}$single cells from the tumors ( $n=2$ ) of MFCinoculated Yap1;Taz ${ }^{D K O}$ mice and control mice (Fig. 5e). After excluding cells of high mitochondrial load (an indicator of cell death) and putative doublets, 10,377 single-cell transcriptomes were retained for analysis and visualized via UMAP. Of all cells, $\sim 56 \%$ of them $(5,847)$ were classified as neutrophils, other cells of them were identified as monocytes and macrophages (2,088), T cells (1,772), NK cells (381), B cells (177) and dendritic cells (112) (Fig. 5f). We observed a decreased percentage of total neutrophil but increased percentage of

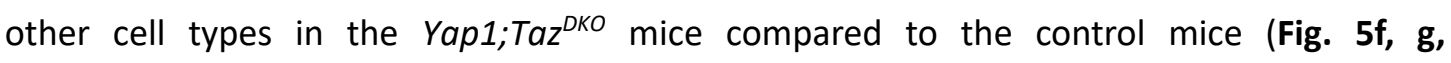
Supplementary Fig. 5d). Consistent with the above observations (Fig. 5c, Supplementary Fig. 5b), YAP/TAZ deficiency led to an obvious decreased percentage of tsNeu3/4 but increased percentage of preNeu (Supplementary Fig. 5e, f). Moreover, tumor-infiltrated neutrophils from Yap1;Taz ${ }^{D K O}$ mice displayed a downregulated transcriptional profile of the activation signature genes like CD14, CD54 (ICAM1), JunD and $N f k b 1$, but an upregulated profile of early lineage signature genes like Hexa, Ly6c2 and Prnt3 (Fig. 5h). Furthermore, KEGG enrichment analysis of $Y a p 1 ; T a z^{D K O}$ versus control mice indicated that the major pathway related to development was Hematopoietic cell lineage, and pathways related to immune activation were the Toll-like receptor signaling, T cell receptor signaling and RIG-I-like receptor signaling (Fig. 5i).

Taken together, these results demonstrate that the YAP/TAZ is essential for the development of tumor-infiltrated neutrophils, and that YAP/TAZ-deficient neutrophils are atavistic and defect in activation.

\section{tsNeu Subsets Are Conserved from Mice to Human}

To determine whether tsNeus were also present in human, we performed inDrop scRNA-seq analysis of $\mathrm{CD} 45^{+}$cells isolated from clinical samples of GC patient (Fig. 6a). The gastric cancer tissue (GC), peritumoral tissue (PT) and peripheral blood (PB) were from one patient who had not yet received cancer treatment. After filtering scRNA-seq data to exclude putative cell 
doublets and stressed or dead cells, a total of 20,985 cell transcriptomes were visualized for exploration via UMAP. Of all cells, $16 \%$ of them $(n=3,357)$ were classified as neutrophils, and other cells of them were identified as monocytes $(1,176)$, macrophages $(1,859), T$ cells $(6,266)$, NK cells $(1,506)$, B cells $(3,969)$ and dendritic cells $(894)$, respectively (Fig. 6b). We then focused on neutrophils, which were resolved into six subsets (immNeu, mNeu, and tsNeu1 4) by spectral clustering (Fig. 6c). Notably, the relative proportions of these neutrophil subsets vary considerably in different tissues: immNeu and mNeu were enriched $\sim 3$ fold in $\mathrm{PB}$ in comparison to PT and GC; tsNeu1/2 was increased $>10$ fold in PT and GC in comparison to PB; tsNeu3/4 were exclusively present in GC (Fig. 6c).

To further define how the neutrophil subsets relate to each other across species, we compared their transcriptional signatures between human and mouse (Fig. 1C, Supplementary Fig. $\mathbf{6 d}$ ). In either human or mouse, tsNeu1/2 expressed high amounts of neutrophil lineage markers (CD63 and CD81) and type I interferon genes (ISG15 and RSAD2), whereas tsNeu3/4 expressed activation markers (ICAM1 and CCL5) (Fig. 1c, 6d). Consistently, tsNeus also showed a highly coordinated expression of tertiary granules (Supplementary Fig. $6 \mathbf{6}, \mathbf{b})$. Regardless of species, the tsNeu3/4 subsets showed higher scores of maturation, aging and apoptosis, compared to tsNeu1/2 (Supplementary Fig. 6c). Moreover, trajectory analysis showed a common ordering of neutrophil subsets from tsNeu1 via tsNeu2, to tsNeu3 and tsNeu4 (Supplementary Fig. 6d). In addition, ligand-receptor interaction analysis ${ }^{44}$, a method to construct a cell-cell affinity matrix revealed high affinity of neutrophils with monocytes in the tumor microenvironment (Fig. 6e).

Our transcriptomic data suggest a similarity in neutrophil development between human and mouse with the identification of human equivalents of tsNeus. To further verify this similarity at the level of protein expression profiles, we next performed flow cytometric analysis of each neutrophil subset. As expected, we observed a clear decrease of $\mathrm{CD} 11 \mathrm{~b}^{+} \mathrm{CD} 66 \mathrm{~b}^{+}$neutrophils along tumor progression (from $47.7 \%$ at stage lla to $4.4 \%$ at stage IIlb)(Fig. 6f-h). Most tumor-infiltrated neutrophils were CD44-CXCR2 in human GC samples, while the majority of neutrophils in the peripheral blood were $\mathrm{CD}_{4} 4^{+} \mathrm{CXCR2} 2^{+}$(Fig. 6f, g). Like murine tsNeus, human tsNeus also consist of four subsets (CD101 CD54, CD101 ${ }^{+} \mathrm{CD} 54^{-}, \mathrm{CD}^{-} \mathrm{10}^{-}$ $\mathrm{CD}^{+} 4^{+}, \mathrm{CD} 101^{+} \mathrm{CD} 54^{+}$) (Fig. 6f, g). Meanwhile, the fractions of CD44-CXCR2 ${ }^{-}, \mathrm{CD} 101^{-} \mathrm{CD}^{-} 4^{+}$and $\mathrm{CD} 101^{+} \mathrm{CD} 54^{+}$cells were decreased, whereas the fraction of $\mathrm{CD} 101^{+} \mathrm{CD} 54^{-}$cells was increased along tumor progression (Fig. 6i).

Taken together, these results indicate that tsNeus are conserved in human and mice, and that the tsNeu subsets were differentially associated with tumor progression.

\section{A Hippo-directed Neutrophil Therapy Inhibits Tumor in Combination with PD-1 Blockage}

Given the enhanced tumor-killing ability of $\mathrm{CD} 54^{+}$tsNeus and our findings that YAP/TAZ is essential for the production and function of such tsNeus, we reasoned that pharmacologic activation of YAP/TAZ may reshape neutrophils for cancer immunotherapy. To test this 
hypothesis, we treated mice with phosphatidic acid (PA), a known Hippo pathway regulator that activates YAP/TAZ via intraperitoneal injections (50,100mg/kg) (Fig. 7a). After injection of PA for 3 consecutive days, we observed an increase in the number of total neutrophils in the murine blood (Fig. 7b). We then isolated neutrophils from the blood of PA-treated mice, found that PA-treatment dose-dependently promoted their tumor-killing ability (Fig. 7c) and the fraction of CD44CXCR2 Neu in blood (Fig. 7d). Consistently, in a nude mice xenograft tumor model, the growth of MFC-derived tumors was significantly inhibited in mice receiving PA treatment when compared to the control group (Fig. 7e). We also observed an increase in the number of total neutrophils in blood (Fig. 7f), as well as the number of $\mathrm{CD} 54^{+}$neutrophils in tumor tissue (Fig. $\mathbf{7 g}$ ). Moreover, tumors-bearing mice adoptively transferred with neutrophils from PA-treated mice showed much smaller tumors when compared to the control group (Fig. 7h).

To further evaluate the immunotherapeutic effects of targeting the Hippo pathway, we isolated $\mathrm{CD} 45^{+} \mathrm{CD} 11 \mathrm{~b}^{+} \mathrm{Ly} 6 \mathrm{G}^{+}$neutrophils from peripheral blood of four GC patients at different tumor stages, as well as Epcam ${ }^{+}$tumor cells from these patients. We then treated the isolated neutrophils with PA for $24 \mathrm{~h}$, and subsequently incubated with tumor cells isolated from the same patient (Supplementary Fig. 7a). We observed that PA treatment strongly increased killing ability of blood neutrophils toward GC of different tumor stage (Fig. 7i). Similar to the observations in mice, PA treatment also significantly increased the percentage of tsNeus (CD44 ${ }^{-}$CXCR2) in human PB neutrophils stimulated with HGC-27, a human GC cell line (Fig. $7 \mathbf{j}$, Supplementary Fig. 7b). Furthermore, we tested a combined therapy of PA and anti-PD-1 antibody in a mouse xenograft tumor model, in which tumors were pre-implanted for 5 days before the treatment. The combined therapy showed a better efficacy than monotherapies in inhibiting tumor progression (Fig. 7k). Note that PA monotherapy had a marginal effect on $\mathrm{CD}^{+} \mathrm{T}$ cells after $\mathrm{CD} 3 / \mathrm{CD} 28$ stimulation (Supplementary Fig. 7c).

Taken together, these results indicate that targeting the Hippo pathway may reprogram neutrophils for cancer immunotherapy, and its combination with anti-PD-1 blockage can further boost the therapeutic effect. 


\section{DISCUSSION}

Due to their complex and context-dependent functions, tumor- associated neutrophils (TANs) remain one class of underestimated components of the tumor microenvironment, especially during the early stage of tumorigenesis. Current views on the opposing roles of neutrophils in cancer is thought to be related to different tumor type and stage of progression. Thus a comprehensive understanding of TANs, in particular their heterogeneity, is urgently needed for the development of neutrophil-based therapeutic strategies against tumors. Recent studies have depicted the transcriptional landscape of neutrophils maturation and fate decision in their steady state and during bacterial infection ${ }^{28,30,33}$. Here, we investigated at both transcriptional and protein levels the signature, function, and heterogeneity of tumorinfiltrated neutrophils in mouse and human gastric cancer. Our study defines CXCR2 ${ }^{\text {neg }} C D 44^{\text {neg }}$ neutrophils as tsNeus, which can be further classified/separated into four subsets according to their expression of CD54 and CD101 (Supplementary Fig. 7d). Molecular dissection of tsNeus identified the Hippo pathway transcriptional coactivators YAP/TAZ as essential factors for the generation and function of teNeus. By pharmacological targeting the Hippo pathway, we show a proof of concept being able to reprogram neutrophils for anti-tumor immunotherapy.

CD101 were previously identified as a marker to distinguish immature neutrophils from mature ones in cancer ${ }^{31}$. In the earliest stages of lung cancer, TANs displayed an activated $\mathrm{CD}^{+} 4^{+}$phenotype and can stimulate $\mathrm{T}$ cell responses ${ }^{11}$. TANs in gastric cancer patients showed an activated $\mathrm{CD} 54^{+}$phenotype and expressed high level immunosuppressive molecule PD-L1 ${ }^{23}$. Here, we found that the expression levels of CD54 in tsNeus were corelated to their tumor cell killing ability, with $\mathrm{CD} 101^{+} \mathrm{CD} 54^{+}$tsNeus showing highest killing ability than other subpopulations. CD54 expression was gradually increased in the early stage of tumor progression in a xenograft tumor model. However, tsNeus from gastric cancer patients of advanced stages showed decreased CD54 expression (tsNeus from stage III GC patients express less CD54 than those from stage II GC patients). Thus, the expression of CD54 was altered along tumor progression, and increasing the amount of $\mathrm{CD} 101^{+} \mathrm{CD} 54^{+}$tsNeus may improve cancer therapy. Our current work identified the combination of CD101 and CD54 as a useful gating strategy to separate or purify four individual tsNeu subsets, paving a way for further study of tsNeus and neutrophil therapy.

Serial transcription factors involved at different stages of neutrophils development have been well characterized ${ }^{32,45}, 46$. Here, we identified YAP/TAZ-TEAD as a key transcriptional reprogramming machinery required for chemoattraction and cytotoxicity of tsNeus. The Hippo-YAP signaling pathway plays a key role in organ size control and tissue homeostasis, and dysregulation of this pathway has been linked to various cancers (Harvey and Tapon, 2007; Steinhardt et al., 2008; Pan, 2010; Zhao et al., 2010; Chan et al., 2011). Despite of extensive efforts devoted to develop anticancer therapies through blocking YAP/TAZ activity in tumor cells, the specific function of YAP/TAZ in immune cell-mediated tumor immunotherapy has not 
been fully elucidated. We found here the percentage of neutrophils (either at baseline or upon tumor engraftment) in bone marrow, spleen or peripheral blood of YAfflox/flox $L y z M^{\text {cre }}$ mice did not differ from those in wildtype control mice. However, deficiency of YAP/TAZ significantly impaired the generation and infiltration of effective tsNeu, therefore accelerating tumor progression. Based on these findings, we designed a strategy of targeting the Hippo-YAP pathway for neutrophil therapy against gastric cancer. Our study demonstrated that enhancing the activity of YAP with the Hippo inhibitor PA can elicit a strong anti-tumor effect of neutrophils. PA treatment in vivo not only increased the number of tsNeus, but also promoted their tumor-killing ability. We also observed significant inhibition of tumor growth by adoptive transfer of PA-treated neutrophils into tumor-bearing mice. This provides a proof of concept that cancer patients could receive PA-treated neutrophils as an immunotherapy.

Myeloid-derived suppressor cells (MDSCs) have emerged as major regulators of immune responses in cancer and other pathological conditions. Polymorphonuclear myeloid- derived suppressor cells (PMN-MDSCs) were originally used to refer to cells with immunosuppressive properties that differentiated and expanded from the immature granulocytes in both mouse models and patients with cancer ${ }^{47}$. Lacking of clear markers to distinguish PMN-MDSC from granulocytes generates confusion in investigation and analysis the function of them in tumor progression. In some tumor models, CD115 may serve as a PMN-MDSCs marker ${ }^{42}$. Our results here showed that $3.8 \%$ of the tumor associated neutrophils were CD115 positive at day 10 of a xenograft tumor model. This suggests that very few neutrophils are PMN-MDSC in the early stages of tumorigenesis. Whether Hippo-YAP signaling plays a role in neutrophils as PMNMDSC warrants further investigation in future.

Interestingly, our study also identified a population of silent neutrophils (sNeu) expressing early lineage markers $\left(\mathrm{CD}^{3} 4^{+} \mathrm{c}-\mathrm{Kit}^{+}\right)$with potential to differentiate into tsNeus, a phenomenon reminiscent of reserved stem cell. In this regard, a preview report proposed that $\mathrm{CD} 34^{+} \mathrm{C}-\mathrm{Kit}^{+}$ early neutrophils with pro-tumoral activity were accumulated in a murine tumor model ${ }^{48}$. The lineage specification of $s \mathrm{Neu}$, as well as their ability to differentiate into tsNeus remain to be clarified. Given the essential roles of YAP/TAZ in tsNeu development, it is also possible that the Hippo-YAP signaling regulates the potential stemness of sNeus and their differentiation into tsNeus.

In summary, our study provides a comprehensive landscape of neutrophils diversity and function in gastric cancer, and identified molecular makers for tumor-specific neutrophils. Moreover, we identified YAP/TAZ as key transcriptional coactivators required for tsNeu fate determination and function. These findings not only elucidate the functional importance of YAP/TAZ regulation of tsNeu, but also showcase a new type of immunotherapeutic approach via pharmacological targeting the Hippo-YAP pathway in neutrophils. 


\section{METHODS}

\section{Cells}

MFC cells were obtained from cell line resource of National Infrastructure (Beijing, China) and were grown in RPMI1640 (Invitrogen/Thermo Fisher Scientific, MA, USA), maintained in culture supplemented with $10 \%$ heat-inactivated FBS (Biological Industries), and 1\% penicillin/streptomycin (Gibco/Thermo) at $37{ }^{\circ} \mathrm{C}$ with $5 \% \mathrm{CO}_{2}$ in a humidified incubator (Thermo). Cells were passaged for $\leq 3$ months from the frozen early-passage stocks that had been received from the indicated sources. During the study, all cell cultures were periodically tested for mycoplasma using MycoAlert ${ }^{\mathrm{TM}}$ Mycoplasma Detection Kits (Lonza, ME, USA).

\section{Mice}

All mice were housed under specific pathogen-free conditions in automated watered and ventilated cages on a 12-hr light/dark cycle and handled in accordance with were the guidelines of the Institutional Animal Care and Use Committee of the Institute of Biochemistry and Cell Biology. The approval ID for the use of animals was SIBCB-NAF-14-004-S329-023 issued by the Animal Core Facility of SIBCB.

Mice used in this study were from SLAC Laboratory Animal (Shanghai, China): 6-week-old $\mathrm{BALB} / \mathrm{c}$ mice (male and female); 6-week-old C57BL/6J mice (male and female). Tumors were allowed to establish, sizes (average $80^{\sim} 120 \mathrm{~mm}^{3}$ ) were matched and then mice were randomly allocated to groups of $6 \sim 10$ animals. No blinding was used in the treatment schedules for these experiments since the different treatments were identified by mark on tail. Based on our previous experience, groups of $6 \sim 10$ animals were used to have sufficient animals per group to provide statistically significant data while keeping the number of animals used to a minimum. Tumor size was determined by caliper measurements of tumor length, width and depth and tumor volume was calculated as volume $=0.5236 \times$ length $\times$ width $\times$ depth $\left(\mathrm{mm}^{3}\right)$.

\section{Murine Tissue Preparation}

All murine tissues in this study were prepared from mice sacrificed by cervical dislocation at the same time. For the isolation of BM cells, leg bones from murine femur were flushed using a 21-gauge needle in RPMI1640 medium containing 1\% FBS. For the isolation of SP cells, the whole spleen were dissected and homogenized into single-cell suspensions using a $1 \mathrm{ml}$ syringe plunger with a 21-gauge needle. For the isolation of PB cells, $600 \sim 800 \mu \mathrm{l}$ blood was collected by retro-orbital bleeding. For the isolation of GC cells, xenograft tumor tissues $\left(300^{\sim} 1,000 \mathrm{~mm}^{3}\right)$ were dissected into 1 2 $\mathrm{mm}$ pieces with scissors in PBS buffer and digested in RPMI 1640 Medium with $1 \mathrm{mg} / \mathrm{ml}$ Collagenase II and $100 \mu \mathrm{g} / \mathrm{ml}$ DNase I at $37^{\circ} \mathrm{C}$ for $30 \mathrm{~min}$.

Subsequently, BM, SP, PB and GC cells were filtered with a $70 \mu \mathrm{m}$ cell strainer (BD Biosciences, San jose, CA). After a 5 min centrifugation at $500 \mathrm{~g}$, cells were resuspended with red blood cell lysis buffer (RT122-02, TIANGEN, Shanghai, China) to deplete red blood cells. 
Subsequently, cells were washed 2 3 times and kept in RMPI1640 medium with 1\% FBS and $2 \mathrm{mM}$ EDTA at $4^{\circ} \mathrm{C}$ before use.

\section{Human GC Sample Preparation}

GC tissues were dissected into 1 2 $\mathrm{mm}$ pieces with scissors in PBS buffer and digested in RPMI 1640 Medium with $1 \mathrm{mg} / \mathrm{ml}$ Collagenase II and $100 \mu \mathrm{g} / \mathrm{ml}$ DNase I at $37^{\circ} \mathrm{C}$ for $30 \mathrm{~min}$. After the depletion of red blood cells. Cells were washed three times and kept in RMPI1640 medium with $1 \% \mathrm{FBS}$ and $2 \mathrm{mM}$ EDTA at $4^{\circ} \mathrm{C}$ before use. GC patients included in the study provided written informed consent for the use of the specimen. The studies were performed in accordance with the Declaration of Helsinki and approved by the Huashan Hospital Institutional Review Board (HIRB), Fudan University (Approval No.2017-222).

\section{Flow Cytometry and Cell Sorting}

Antibodies were purchased from BD, Biolegend, eBioscience or Thermo Fisher (Table SXX). For the identification of preNit1/2 and Nit1/2 Neu, cells were blocked with anti-mouse antibodies against CD16/32 (HI30) for $20 \mathrm{~min}$ at $4^{\circ} \mathrm{C}$. After exclusion of cell doublets and dead cells with LIVE/DEAD, cells were stained with fluorophore-conjugated anti-mouse antibodies against CD45 (30-F11), CD11b (M1/70), Ly6G (1A8), Ly6C (M1/70), CD54 (YN1/1.7.4), and $\mathrm{CD} 101$ (Moushi101) for $30 \mathrm{~min}$ at $4^{\circ} \mathrm{C}$ (Table S4). Flow cytometry acquisition was performed on a 4-laser BD LSR Fortessa ${ }^{\mathrm{TM}}$ (BD) using FACSDiva software, and data was subsequently analyzed with FlowJo software (Tree three).

For the sorting of murine and human cells, cells $\left(2 \times 10^{7} / \mathrm{ml}\right)$ were incubated with the indicated fluorophore-conjugated antibodies for $30 \mathrm{~min}$ at $4^{\circ} \mathrm{C}$. After exclusion of cell doublets and dead cells with DAPI, single cells were purified by fluorescence-activated cell sorting (FACS) using a FACS Arialll cell sorter and Diva software (both BD Biosciences) to achieve > $98 \%$ purity.

\section{Neu Killing Assay}

Murine MFC cells were seeded at 3,000 cells/well in 96-well microtiter plates with $1001616 \mu \mathrm{l}$ culture medium for $24 \mathrm{~h}$ and then cocultured with FACS-purified murine Neu $(9,000$ cells/well) for the following $48 \mathrm{~h}$. An ATP-based CellTilter-LumiTM Plus cell viability assay kit (Beyotime) was used for detecting tumor-killing ability of Neu. $100 \mu \mathrm{l}$ of the reagent was added into each individual well and mixed for $10 \mathrm{~min}$ at room temperature, then intracellular ATP content was measured using a luminescence detector (GloMax ${ }^{\circledR} 20 / 20$, Promega). Killing ability of Neu was calculated using the following equation: \% Killing ability $=100-$ [value (test) - value (blank)] $\times$ [value (control) - value (blank) $]^{-1} \times 100$.

\section{Murine Strains}

Yap1 ${ }^{\text {Floxed }}$; Taz $^{\text {Floxed }}$ mice has been described (XX). Genomic DNA extracted from tail biopsies was used to evaluate offspring genotype. $L y s M^{c r e}$ mice were purchased from Jackson. $L y s M^{\text {cre }}$ mice

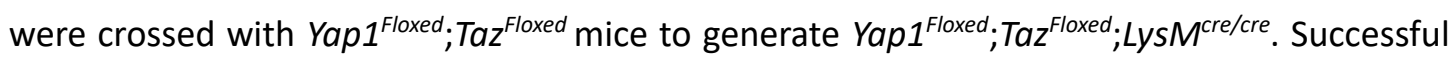
YAP/TAZ deletion in Neu was confirmed by immunoblotting. 


\section{Single Cell RNA (sc-RNA) Sequencing}

Designated cells were sorted into PBS containing 0.05\% BSA following the 10x Genomics protocol. The cell preparation time before loading onto the $10 \times$ Chromium controller was $<2 \mathrm{~h}$. Cell viability and counting were evaluated with trypan blue by microscopy, and samples with viabilities $>85 \%$ were used for sequencing. Libraries were constructed using the Single Cell $3^{\prime}$ Library Kit V2 (10x Genomics). Transcriptome profiles of individual cells were determined by 10x Genomics-based droplet sequencing. Once prepared, indexed complementary DNA (cDNA) libraries were sequenced with paired-end reads on an Illumina NovaSeq 6000 (Illumina). The GEO accession number for the high-throughput sequencing reported in this paper are GSE168537.

\section{Immunoblotting}

Cell lysates were resolved in SDS-PAGE gels. The proteins were transferred to a PVDF membrane (Bio-Rad) and further incubated with antibodies.

\section{Statistical Analysis}

Both cellular and animal studies have tended to be underpowered. Estimation of sample size for planned comparisons of two independent means using a two-tailed test were undertaken using the SAS statistical software package (9.1.3). Data are presented as mean \pm S.D. for continuous variables and as frequencies and proportions for categorical variables. Continuous data were compared using Student's $t$-tests (comparing two variables) or one-way ANOVA analysis (comparing multiple variables). Two to three biological replicates were used throughout the study.

\section{CONFLICT of INTERESTS}

The authors declare no conflict of interest.

\section{ACKNOWLEDGEMENTS}

This work was supported by the National Key R\&D Program of China (2020YFA0803200 and 2017YFA0504504), National Natural Science Foundation of China Grants (81725014, $81822035,81902806,81902389$, and 82002493), the Youth Innovation Promotion Association of the Chinese Academic Sciences, the "Strategic Priority Research Program" (XDB19020202 and XDA12010315), the Key Program for Basic Research of Shanghai (19JC1415600), Shanghai Pujiang Program (19PJ1408300), and the China Postdoctoral Science Foundation (2020T130477).

\section{AUTHOR CONTRIBUTIONS}

PN, SJ and ZZ conceived the ideas and designed the experiments. PN, WZ, YM, FG, HZ and ZT performed most of experiments. MW, FC, LA, YH, WW and LW discussed and analyzed the data. $\mathrm{PN}, \mathrm{WZ}, \mathrm{SJ}$ and $\mathrm{ZZ}$ wrote the manuscript. 


\section{REFERENCES}

1. Kolaczkowska, E. \& Kubes, P. Neutrophil recruitment and function in health and inflammation. Nature reviews. Immunology 13, 159-175 (2013).

2. Shaul, M.E. \& Fridlender, Z.G. Tumour-associated neutrophils in patients with cancer. Nat Rev Clin Oncol 16, 601-620 (2019).

3. Jaillon, S. et al. Neutrophil diversity and plasticity in tumour progression and therapy. Nat Rev Cancer 20, 485-503 (2020).

4. Finisguerra, V. et al. MET is required for the recruitment of anti-tumoural neutrophils. Nature 522, 349-353 (2015).

5. Gershkovitz, M. et al. TRPM2 Mediates Neutrophil Killing of Disseminated Tumor Cells. Cancer research 78, 2680-2690 (2018).

6. Blaisdell, A. et al. Neutrophils Oppose Uterine Epithelial Carcinogenesis via Debridement of Hypoxic Tumor Cells. Cancer Cell 28, 785-799 (2015).

7. Granot, Z. et al. Tumor entrained neutrophils inhibit seeding in the premetastatic lung. Cancer Cell 20, 300-314 (2011).

8. Triner, D. et al. Neutrophils Restrict Tumor-Associated Microbiota to Reduce Growth and Invasion of Colon Tumors in Mice. Gastroenterology 156, 1467-1482 (2019).

9. Dmitrieva-Posocco, O. et al. Cell-Type-Specific Responses to Interleukin-1 Control Microbial Invasion and Tumor-Elicited Inflammation in Colorectal Cancer. Immunity 50, 166-180.e167 (2019).

10. Singhal, S. et al. Origin and Role of a Subset of Tumor-Associated Neutrophils with AntigenPresenting Cell Features in Early-Stage Human Lung Cancer. Cancer Cell 30, 120-135 (2016).

11. Eruslanov, E.B. et al. Tumor-associated neutrophils stimulate T cell responses in early-stage human lung cancer. The Journal of clinical investigation 124, 5466-5480 (2014).

12. Ponzetta, A. et al. Neutrophils Driving Unconventional T Cells Mediate Resistance against Murine Sarcomas and Selected Human Tumors. Cell 178, 346-360.e324 (2019).

13. Mensurado, S. et al. Tumor-associated neutrophils suppress pro-tumoral IL-17+ $\gamma \delta \mathrm{T}$ cells through induction of oxidative stress. PLoS Biol 16, e2004990 (2018).

14. Shojaei, F., Singh, M., Thompson, J.D. \& Ferrara, N. Role of Bv8 in neutrophil-dependent angiogenesis in a transgenic model of cancer progression. Proc Natl Acad Sci USA 105, 2640 (2008).

15. Nozawa, H., Chiu, C. \& Hanahan, D. Infiltrating neutrophils mediate the initial angiogenic switch in a mouse model of multistage carcinogenesis. Proc Natl Acad Sci USA 103, 12493 (2006).

16. Albrengues, J. et al. Neutrophil extracellular traps produced during inflammation awaken dormant cancer cells in mice. Science 361, eaao4227 (2018).

17. Szczerba, B.M. et al. Neutrophils escort circulating tumour cells to enable cell cycle progression. Nature 566, 553-557 (2019).

18. Cools-Lartigue, J. et al. Neutrophil extracellular traps sequester circulating tumor cells and promote metastasis. The Journal of clinical investigation 123, 3446-3458 (2013).

19. Yang, L. et al. DNA of neutrophil extracellular traps promotes cancer metastasis via CCDC25. Nature 583, 133-138 (2020).

20. Spiegel, A. et al. Neutrophils Suppress Intraluminal NK Cell-Mediated Tumor Cell Clearance and Enhance Extravasation of Disseminated Carcinoma Cells. Cancer discovery 6, 630-649 (2016).

21. Coffelt, S.B. et al. IL-17-producing $\gamma \delta$ T cells and neutrophils conspire to promote breast cancer 
metastasis. Nature 522, 345-348 (2015).

22. Veglia, F. et al. Fatty acid transport protein 2 reprograms neutrophils in cancer. Nature $569,73-$ 78 (2019).

23. Wang, T.T. et al. Tumour-activated neutrophils in gastric cancer foster immune suppression and disease progression through GM-CSF-PD-L1 pathway. Gut 66, 1900-1911 (2017).

24. Ng, L.G., Ostuni, R. \& Hidalgo, A. Heterogeneity of neutrophils. Nature reviews. Immunology 19, 255-265 (2019).

25. Ballesteros, I. et al. Co-option of Neutrophil Fates by Tissue Environments. Cell (2020).

26. Akashi, K., Traver, D., Miyamoto, T. \& Weissman, I.L. A clonogenic common myeloid progenitor that gives rise to all myeloid lineages. Nature 404, 193-197 (2000).

27. Németh, T., Sperandio, M. \& Mócsai, A. Neutrophils as emerging therapeutic targets. Nat Rev Drug Discov 19, 253-275 (2020).

28. Xie, X. et al. Single-cell transcriptome profiling reveals neutrophil heterogeneity in homeostasis and infection. Nat Immunol 21, 1119-1133 (2020).

29. Dinh, H.Q. et al. Coexpression of CD71 and CD117 Identifies an Early Unipotent Neutrophil Progenitor Population in Human Bone Marrow. Immunity 53, 319-334.e316 (2020).

30. Kwok, I. et al. Combinatorial Single-Cell Analyses of Granulocyte-Monocyte Progenitor Heterogeneity Reveals an Early Uni-potent Neutrophil Progenitor. Immunity 53, 303-318 e305 (2020).

31. Evrard, M. et al. Developmental Analysis of Bone Marrow Neutrophils Reveals Populations Specialized in Expansion, Trafficking, and Effector Functions. Immunity 48, 364-379.e368 (2018).

32. Hock, H. et al. Intrinsic requirement for zinc finger transcription factor Gfi-1 in neutrophil differentiation. Immunity 18, 109-120 (2003).

33. Evrard, M. et al. Developmental Analysis of Bone Marrow Neutrophils Reveals Populations Specialized in Expansion, Trafficking, and Effector Functions. Immunity 48, 364-379 e368 (2018).

34. Xie, X. et al. Single-cell transcriptome profiling reveals neutrophil heterogeneity in homeostasis and infection. Nat Immunol 21, 1119-1133 (2020).

35. Qiu, X. et al. Reversed graph embedding resolves complex single-cell trajectories. Nat Methods 14, 979-982 (2017).

36. Borregaard, N., Sorensen, O.E. \& Theilgaard-Monch, K. Neutrophil granules: a library of innate immunity proteins. Trends Immunol 28, 340-345 (2007).

37. Cowland, J.B. \& Borregaard, N. Granulopoiesis and granules of human neutrophils. Immunol Rev 273, 11-28 (2016).

38. Beinert, T., Münzing, S., Possinger, K. \& Krombach, F. Increased expression of the tetraspanins CD53 and CD63 on apoptotic human neutrophils. Journal of leukocyte biology 67, 369-373 (2000).

39. Metkar, S., Kim, K.S., Silver, J. \& Goyert, S.M. Differential expression of CD14-dependent and independent pathways for chemokine induction regulates neutrophil trafficking in infection. Journal of leukocyte biology 92, 389-396 (2012).

40. van der Maaten, L. \& Hinton, G. Visualizing data using t-SNE. J Mach Learning Res 9, 25792605 (2008).

41. Huang, B. et al. Gr-1+CD115+ immature myeloid suppressor cells mediate the development of tumor-induced T regulatory cells and T-cell anergy in tumor-bearing host. Cancer research 66, 
1123-1131 (2006).

42. Youn, J.I., Collazo, M., Shalova, I.N., Biswas, S.K. \& Gabrilovich, D.I. Characterization of the nature of granulocytic myeloid-derived suppressor cells in tumor-bearing mice. Journal of leukocyte biology 91, 167-181 (2012).

43. Aibar, S. et al. SCENIC: single-cell regulatory network inference and clustering. Nat Methods 14, 1083-1086 (2017).

44. Ren, X. et al. Reconstruction of cell spatial organization from single-cell RNA sequencing data based on ligand-receptor mediated self-assembly. Cell Res 30, 763-778 (2020).

45. Yamanaka, R. et al. Impaired granulopoiesis, myelodysplasia, and early lethality in CCAAT/enhancer binding protein epsilon-deficient mice. Proc Natl Acad Sci U S A 94, 1318713192 (1997).

46. Borregaard, N. Neutrophils, from marrow to microbes. Immunity 33, 657-670 (2010).

47. Ostrand-Rosenberg, S. \& Sinha, P. Myeloid-derived suppressor cells: linking inflammation and cancer. J Immunol 182, 4499-4506 (2009).

48. Zhu, Y.P. et al. Identification of an Early Unipotent Neutrophil Progenitor with Pro-tumoral Activity in Mouse and Human Bone Marrow. Cell Rep 24, 2329-2341 e2328 (2018). 


\section{FIGURE LEGENDS}

a

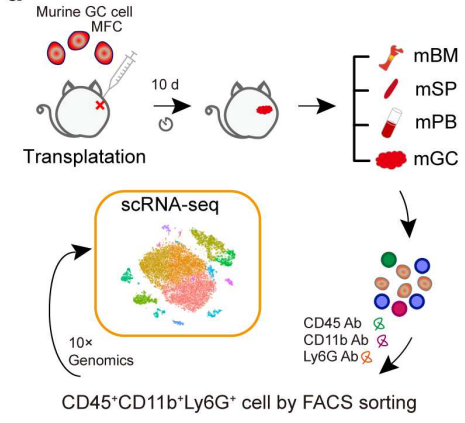

b

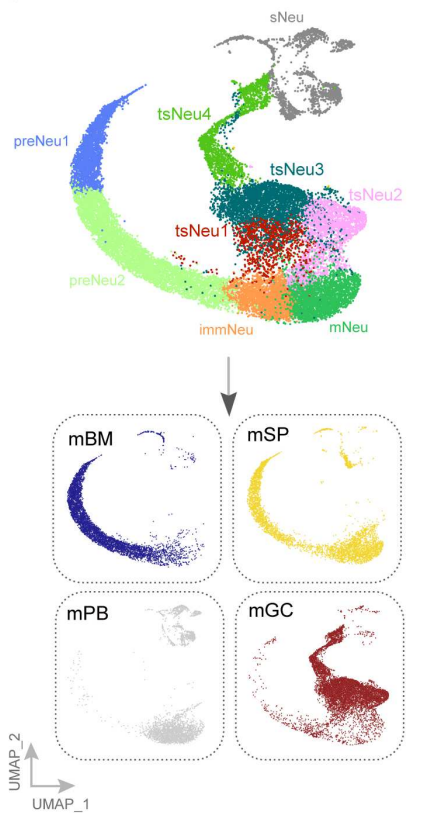

c

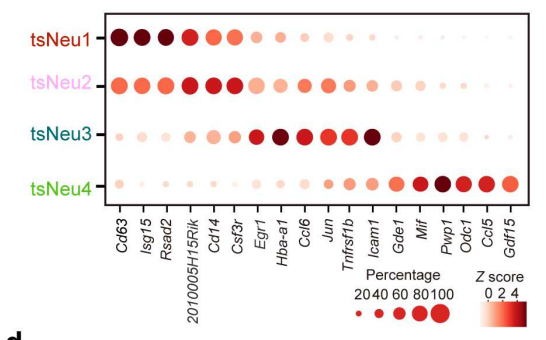

d

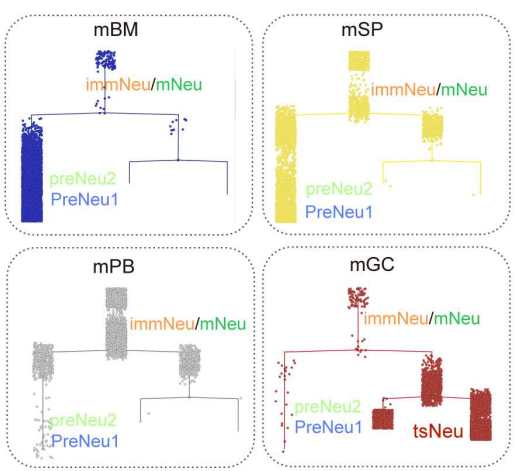

g
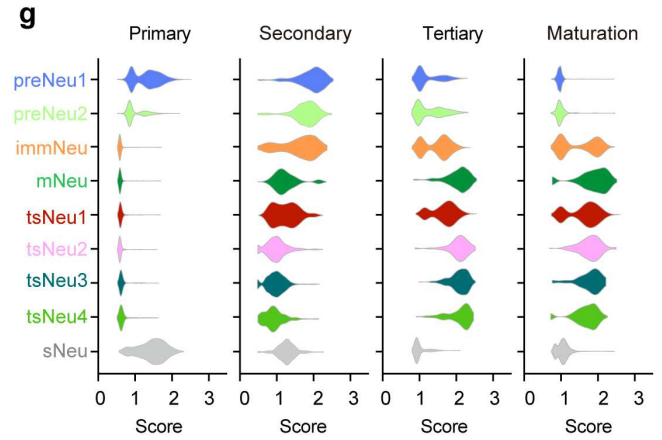

e
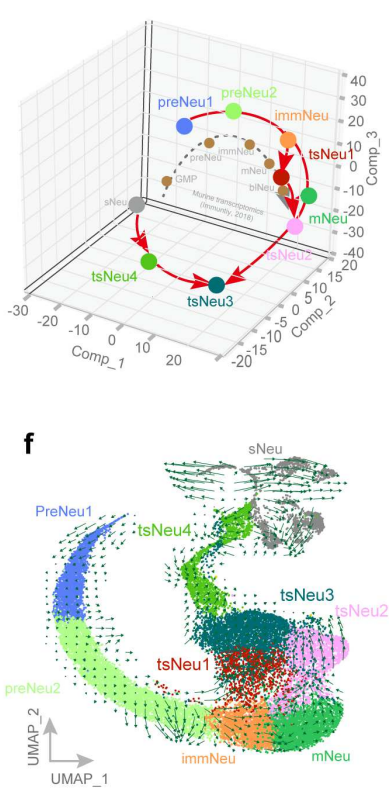

h

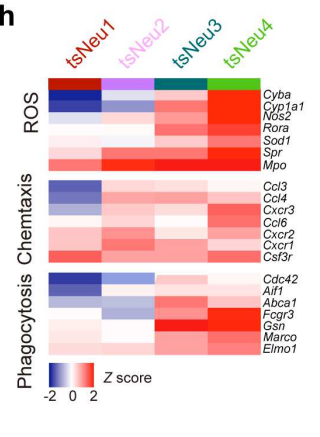

Figure 1 scRNA Sequencing Reveals Tumor-specific Neu (tsNeu) with Distinct Transcriptomic Signatures. (a) Schematic of experimental workflow. Murine $C D 45^{+} C D 11 b^{+} L y 6 G^{+}$cells from bone marrow $(\mathrm{mBM})$, spleen $(\mathrm{mSP})$, peripheral blood $(\mathrm{mBL})$ and $\mathrm{GC}$ tumor $(\mathrm{mGC})$ were sorted by FACS and profiled by 10xGenomics-based scRNA-seq. (b) Uniform Manifold Approximation and Projection (UMAP) plots of 22,069 Neu from mBM, mSP, mPB and mGC identified by integrated analysis, colored by cluster (upper) and tissue (below). preNeu1/2, pre-Neu 1/2; immNeu, immature Neu; mNeu, mature Neu; tsNeu1 4, tumor-specific Neu 1 4; sNeu, silence Neu. (c) Dot plot showing the scaled expression of selected signature genes for the indicated cell clusters (tsNeu1 4 ), colored by the average expression of each gene in each cluster scaled across all clusters. Dot size represents the percentage of cells in each cluster with more than one read of the corresponding gene. (d) Monocle trajectory prediction for the sequenced neutrophils from the indicated samples. (e) Principal-component analysis (PCA) of gene expression data from Neu subsets and available bulk RNA-seq. (f) Velocity analysis revealing the inter-relationship of Neu subpopulations. Velocity fields were projected onto the UMAP plot. (g) Violin plots of primary (azurophil) score, secondary (specific) score, teriary (gelatinase score and secretory) score and mature score for the Neu clusters. (h) Heatmap showing the 
expression of ROS biosynthetic-process-, phagocytosis- and chemotaxis-related genes in the indicated Neu subsets. See also Supplementary Figure 1 

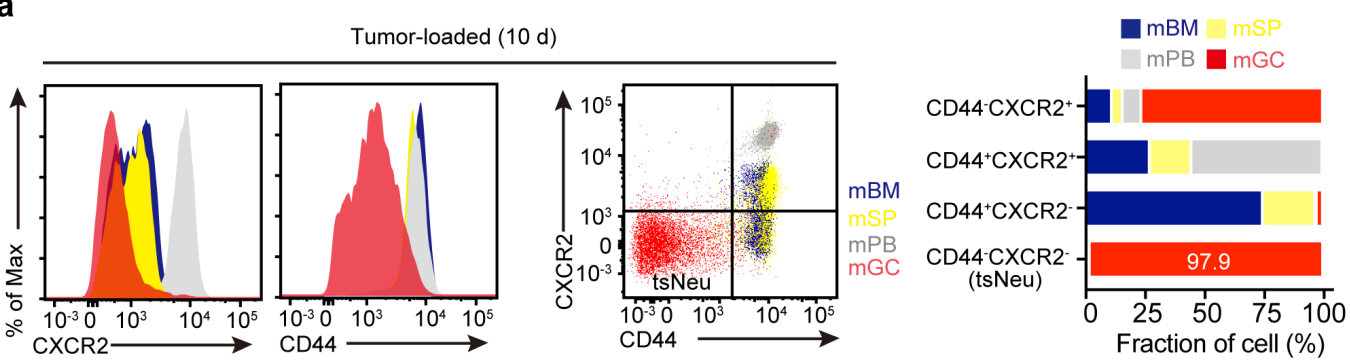

b

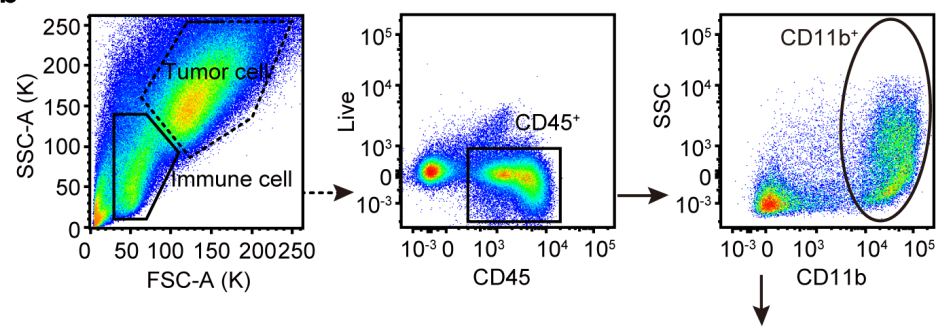

C
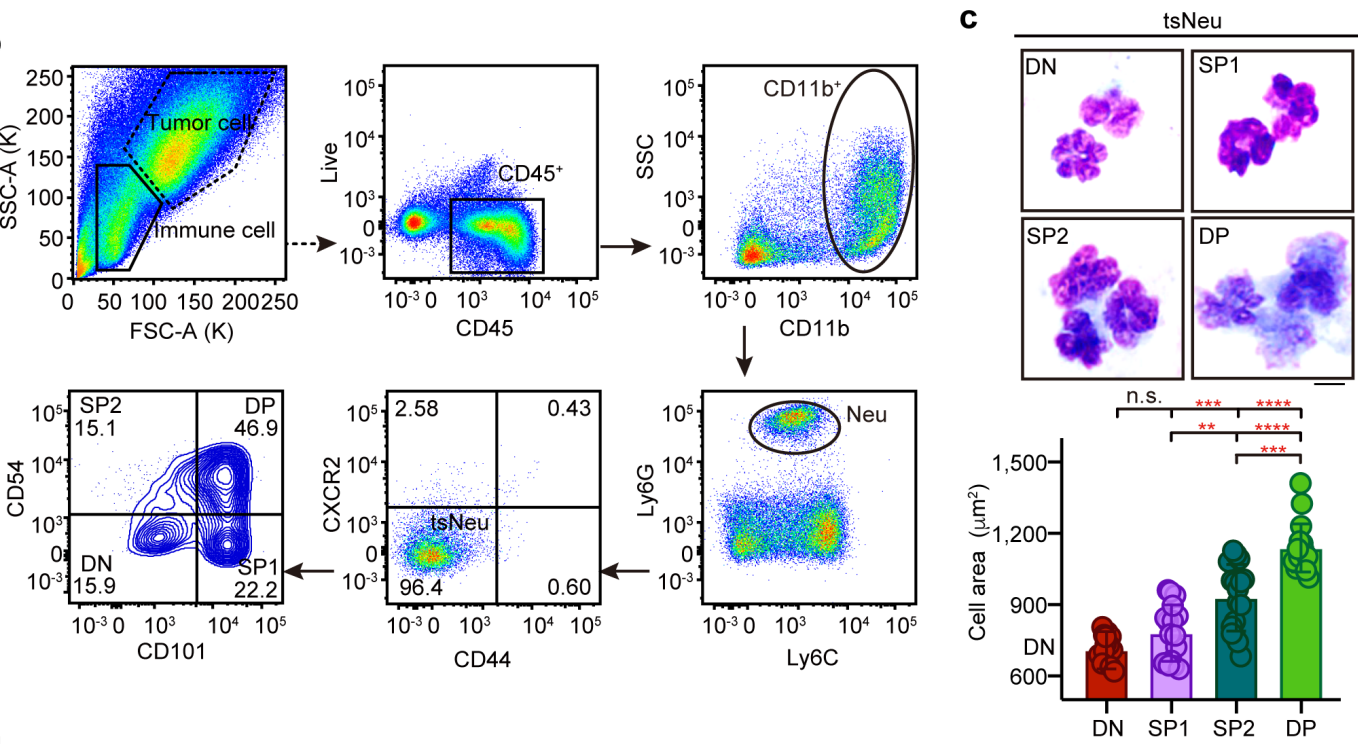

d

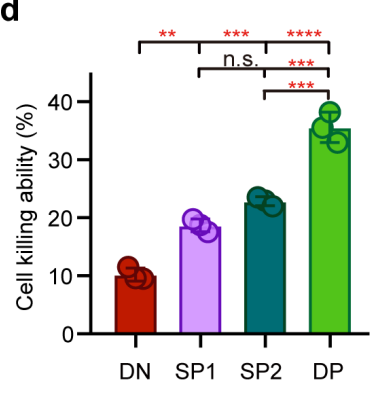

e

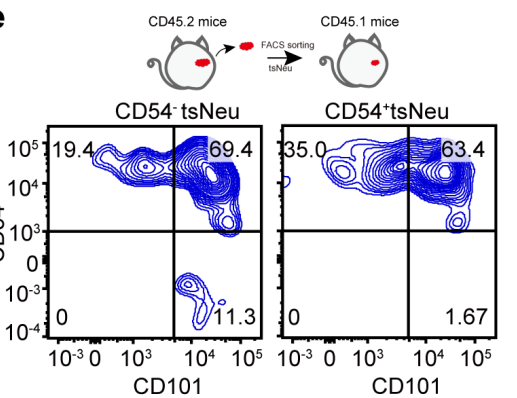

f

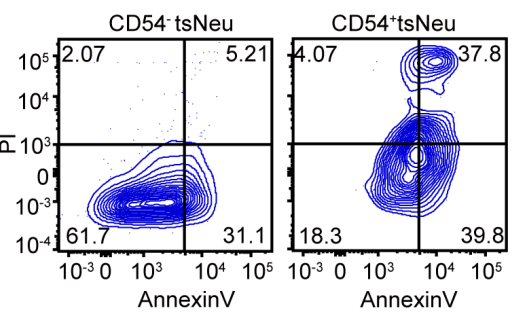

Figure 2 Flow Cytometry Identified A CD44 ${ }^{\text {neg }} C X C R 22^{\text {neg }}$ tsNeu Subpopulation with Four Distinct Subpopulations. (a) Expression of CD44 and CXCR2 among Ly6G ${ }^{+}$Neu from BM (blue), spleen (yellow), peripheral blood (gray) and GC (red). Bar graph showing the frequency of CD44$\mathrm{CXCR}^{-}, \mathrm{CD}_{4}{ }^{-} \mathrm{CXCR} 2^{+}, \mathrm{CD} 44^{+} \mathrm{CXCR} 2^{-}$, and $\mathrm{CD} 44^{+} \mathrm{CXCR} 2^{+}$subsets in four tissues. (b) Representative FACS plots showing the gating strategy of GC Neu. (c) Representative images of sorted subpopulations indicated in (b). The stained areas of cell were quantified by Fuji ImageJ software $(n=20)$. Scale bars, $10 \mu \mathrm{m}$. (d) Killing ability of the indicated tsNeu subsets cocultured with gastric tumor cells (MFC). Cytotoxicity was determined by LDH release assay. (e) Transfer of sorted CD45.2 $2^{+}$tsNeu1/2 or CD45.2 $2^{+}$tsNeu3/4 into CD45.1 $1^{+}$wildtype recipients. Results represent transferred subset after 2 days. (f) Apoptosis of sorted CD54-tsNeu or CD54

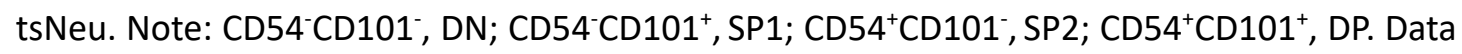
in Figure 2C and 2D are analyzed by one way ANOVA, , followed by Tukey's post-hoc test. **, 
$\mathrm{p}<0.01 ;{ }^{* *}, \mathrm{p}<0.001 ;{ }^{* * *}, \mathrm{p}<0.0001 ; \mathrm{n}$.s., no significance in comparison with control group (same below). See also Supplementary Figure 2 
a

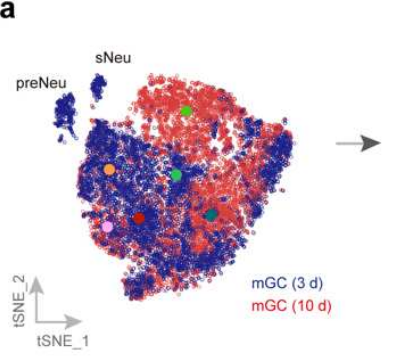

C

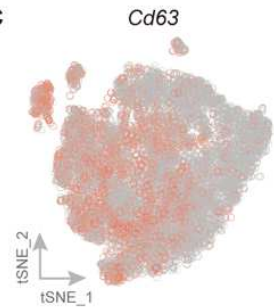

e

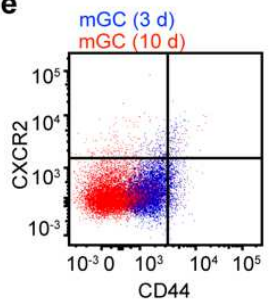

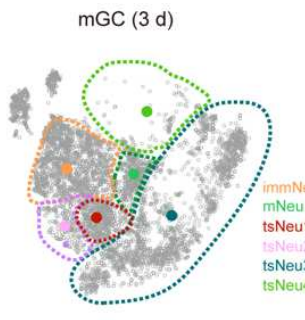

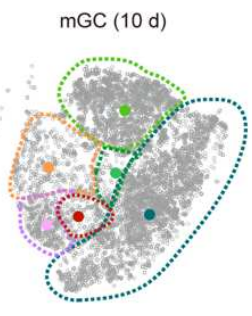

b

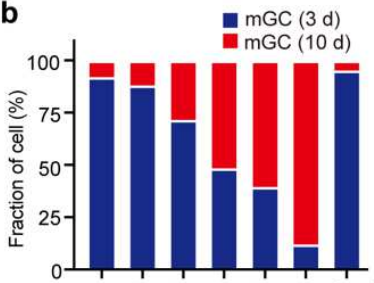

Icam1
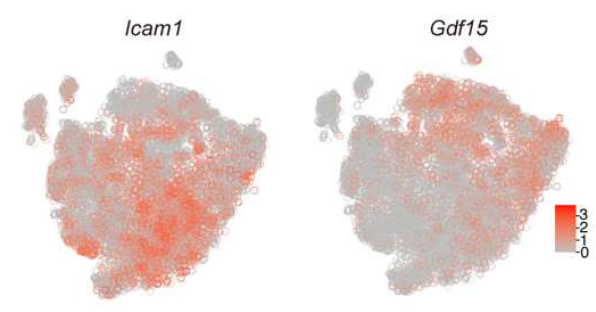

d
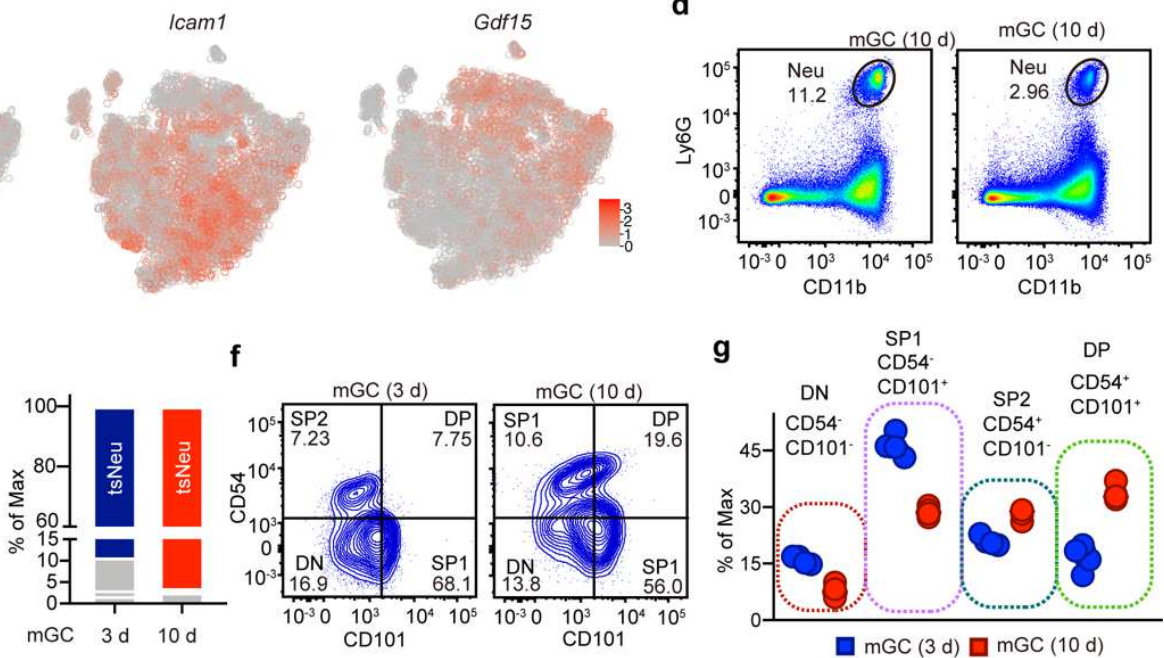

f

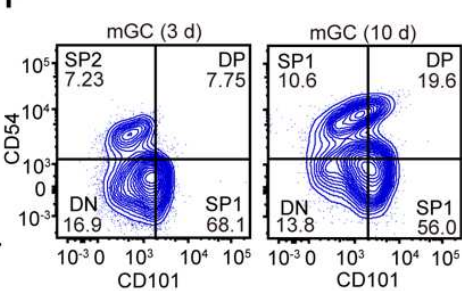

Figure 3 Dynamic Change of tsNeu During Tumor Progression. (a) t-SNE plots of CD11 $\mathrm{b}^{+} \mathrm{Ly} 6 \mathrm{G}^{+}$ Neu from tumor-bearing mice. (b) Bar graph showing the frequency of the indicated subsets from mice bearing tumors for 3 days or 10 days. (c) Single-cell gene expression for representative subsets-enriched genes in $\mathrm{CD} 11 \mathrm{~b}^{+} \mathrm{Ly}_{6 \mathrm{G}}{ }^{+} \mathrm{Neu}$. (d) Flow cytometric analysis of CD11 b ${ }^{+} \mathrm{Ly} 6 \mathrm{G}^{+} \mathrm{Neu}$ from tumor-bearing mice. (e) Expression of CD44 and CXCR2 among CD11 b ${ }^{+} \mathrm{Ly}_{6 \mathrm{G}}{ }^{+}$Neu from mice after subcutaneous implantation with MFC cells for 3 days (blue) or 10 days (red). Bar graph showing the frequency of tsNeu (CD44 ${ }^{-} X C R 2^{-}$) in murine tumor ( $n=6)$. (f) Expression plots of CD54 and CD101 denoting the various tsNeu subpopulations. (g) Scatter plot showing the frequency of the various tsNeu subpopulations. See also Supplementary Figure 3 

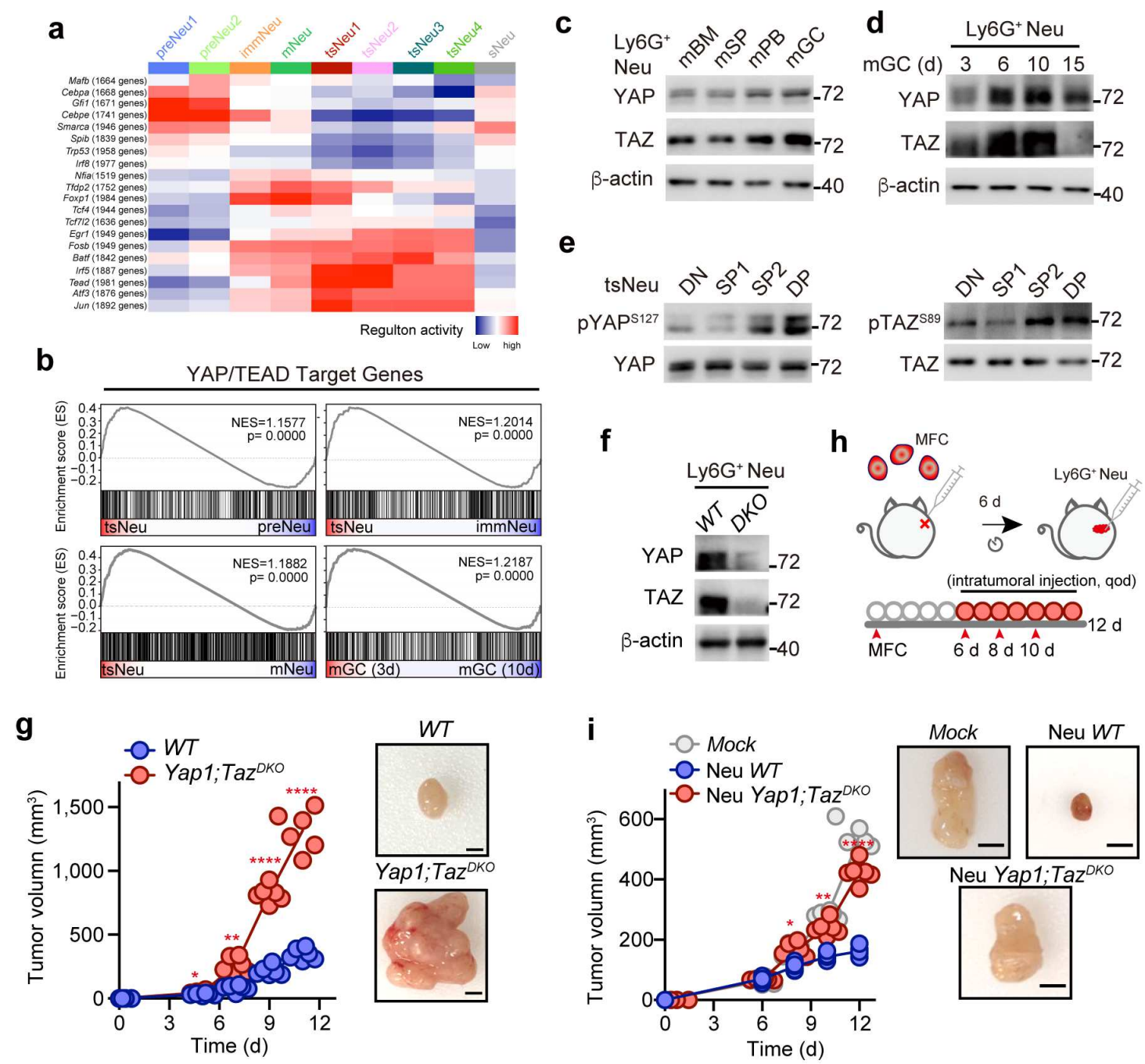

Figure 4 Transcriptomic Analysis Reveals Hippo/TEADs Signaling During tsNeu Development. (a) Heatmap of regulon activity derived from different Neu subsets. Only regulons with $t$ value $>100$ are visualized. (b) GSEA analysis showing significant enrichment of Hippo/TEADs signatures in tsNeu subsets. (c) Immunoblotting showing the expression of YAP and TAZ in total Neu from the indicated tissues. (d) Immunoblotting showing the expression of YAP and TAZ in total $\mathrm{Ly}_{6 \mathrm{G}}{ }^{+} \mathrm{Neu}$ from mic bearing tumors for the indicated periods. (e) Immunoblotting showing the expression of pYAP ${ }^{5127}$, pTAZ $^{589}$, YAP and TAZ in different tsNeu subsets. (f)

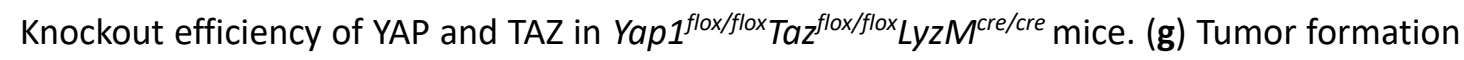

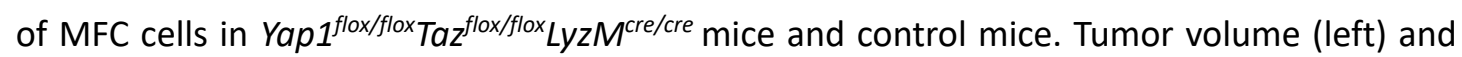
representative photographs (right) are shown. Scale bars, $5 \mathrm{~mm}$. (h) Experimental workflow of tumor formation assay after transferring tsNeu cells to tumor. (i) Transferring assay of Neu to MFC-induced tumor. Tumor volume (left) and representative photographs (right) are shown. Scale bars, $5 \mathrm{~mm}$. See also Supplementary Figure 4 


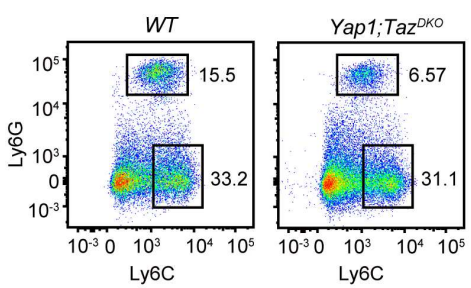

e

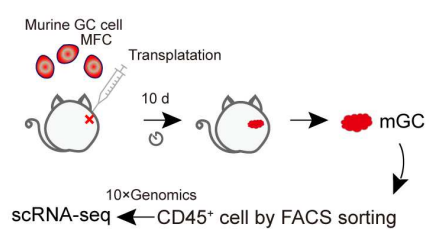

f

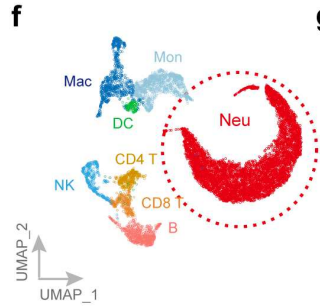

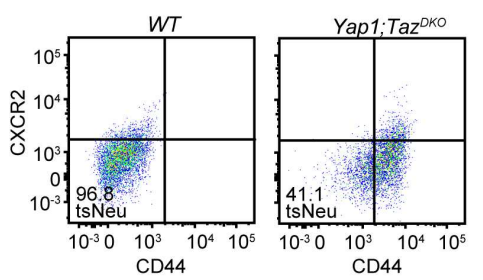

h

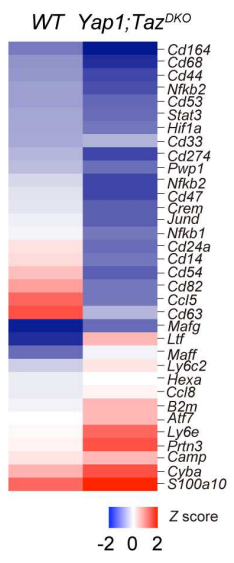

d
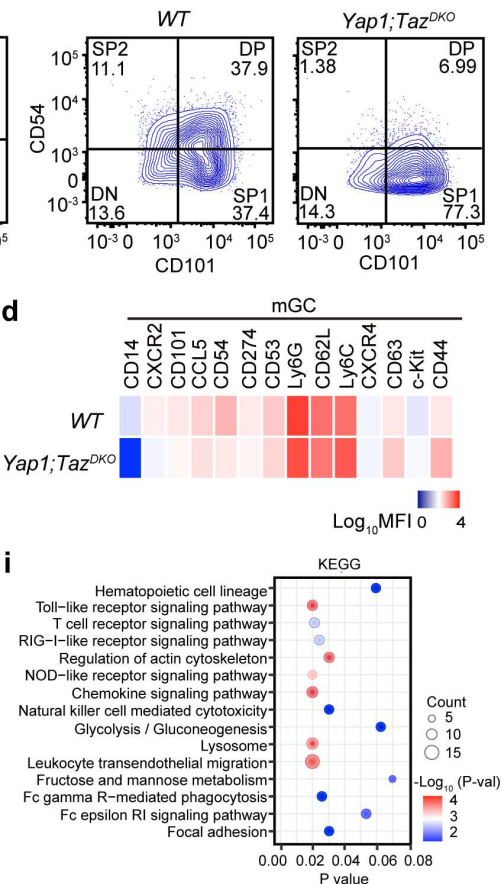

Figure 5 YAP/TAZ Is Pivotal for tsNeu Development. (a) Flow cytometric analysis of $\mathrm{CD} 11 \mathrm{~b}^{+} \mathrm{Ly}_{6 \mathrm{G}}{ }^{+} \mathrm{Ly} 6 \mathrm{C}^{10} \mathrm{Neu}$ in the tumor samples from the Yap1 ${ }^{\text {flox/flox }} \mathrm{Taz}{ }^{\text {flox/flox }} \mathrm{LyzM}^{\text {cre/cre }}$ mice after subcutaneous implantation with MFC cells for 10 days. (b,c) Flow cytometric plots of tsNeus and their subsets from murine tumor samples. (d) Heatmap showing the expression of the indicated markers in the tsNeus from murine tumor tissues. (e) Workflow of the scRNA-seq on murine $\mathrm{CD} 45^{+}$cells from the tumor samples. (f) UMAP plots of $10,377 \mathrm{CD} 45^{+}$cells from the murine tumor. (g) Bar graph showing the frequency of the indicated subsets. (h) Heatmap showing the transcription of Neu-related genes in $\mathrm{Neu}$ clusters from Yap1 $1^{\text {flox/flox }} T a z^{\text {flox/flox }} L y z M^{\text {cre/cre }}$ mice and control mice. (I) KEGG analysis showing top 15 enriched pathway in the Yap $1^{\text {flox/flox }} \mathrm{Taz}^{\text {flox/flox }} \mathrm{LyzM}^{\text {cre/cre }}$ mice versus control mice. See also Supplementary Figure 5. 
a

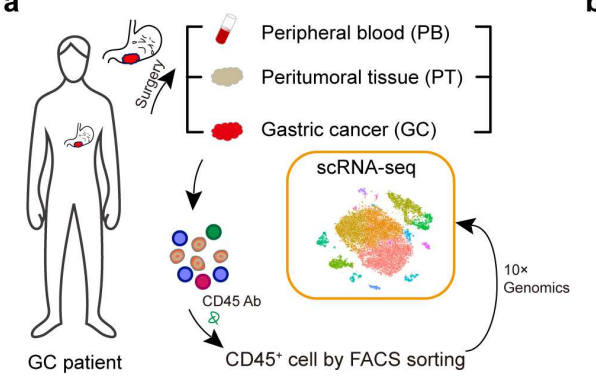

C

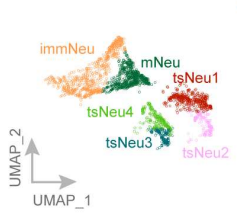

f GC (lla stage)
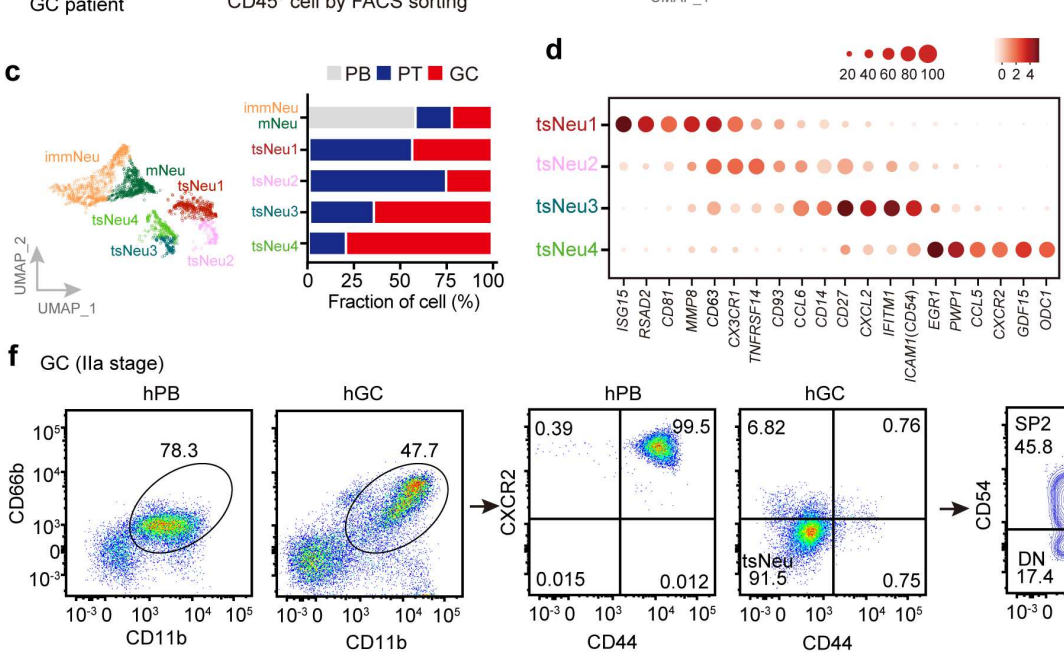

CD44

g GC (IIlb stage)
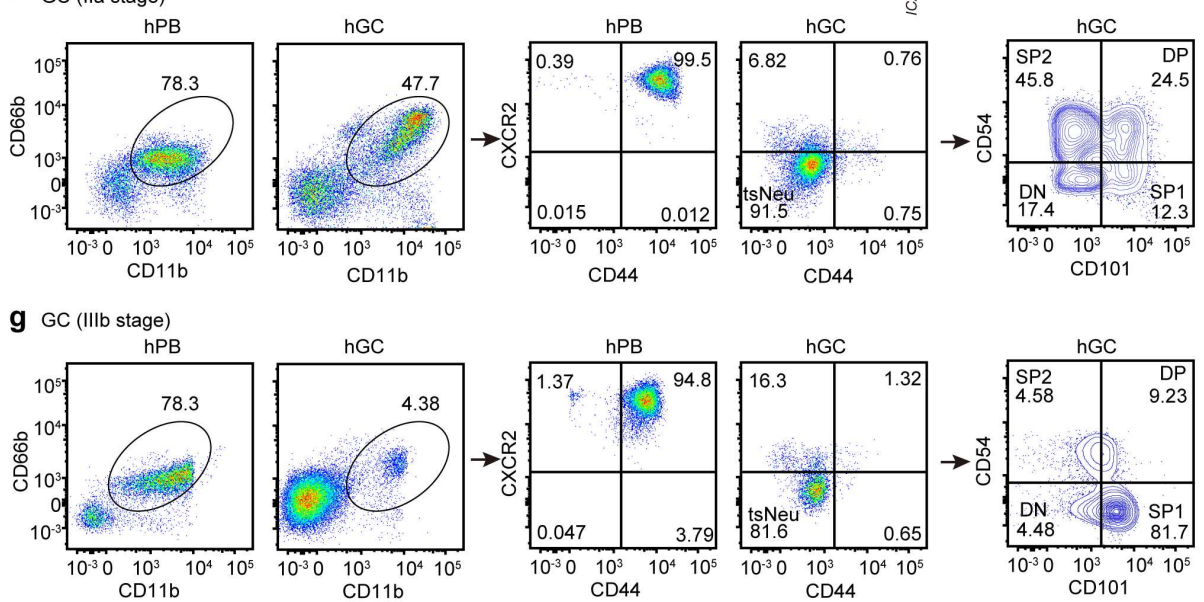

CD101
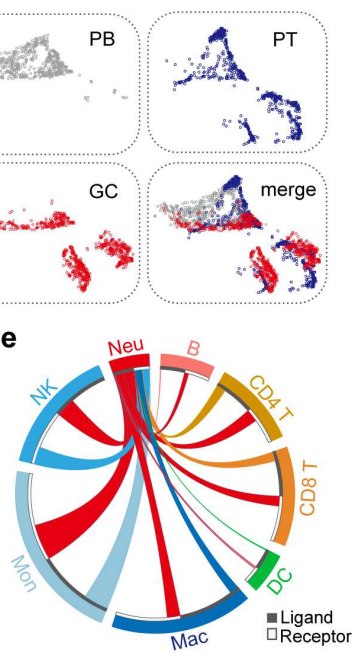

h

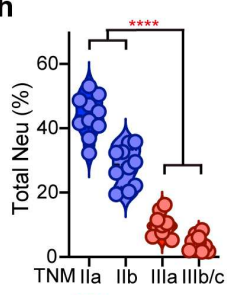

SP1

$100-1 \mathrm{DN}$ SP1 SP2 DP

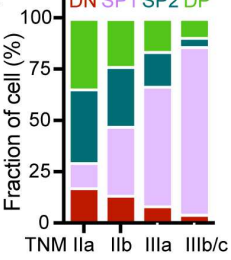

Figure 6 tsNeu Are Present in both Human and Mice and Are Associated with Tumor Progression. (a) Experimental workflow. FACS-isolated $C D 45^{+}$cells from the peripheral blood $(\mathrm{PB})$, peritumoral tissue $(\mathrm{PT})$ and gastric cancer tissue from GC patient and profiled by 10xGenomics-based scRNA-seq. (b) UMAP plots of total CD45 immune cells colored by cluster (left) and tissue (right). (c) UMAP plot showing Neu subsets. Bar graph showing the distribution of the indicated tsNeu subsets among three samples. (d) Dot plot showing the expression of selected signature genes for the indicated tsNeu clusters. (e) Circus plot showing ligand-receptor interactions between Neu and other immune cell types. $(\mathbf{f}, \mathbf{g})$ Gating strategy used to identify tsNeu populations from human GC patients. (h) Violin plot showing the frequency of $\mathrm{CD} 11 \mathrm{~b}^{+} \mathrm{CD} 66 \mathrm{~b}^{+} \mathrm{Neu}$ in the GC tissues (i) Bar graph showing the distribution of tsNeu populations in the GC tissues. See also Supplementary Figure 6. 
a

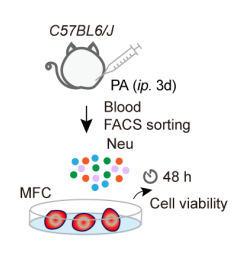

e

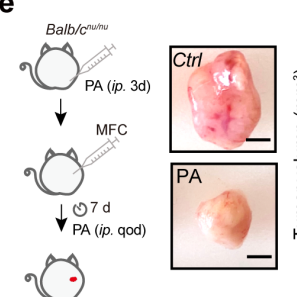
8

h

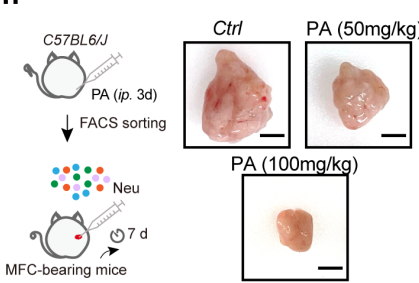

b
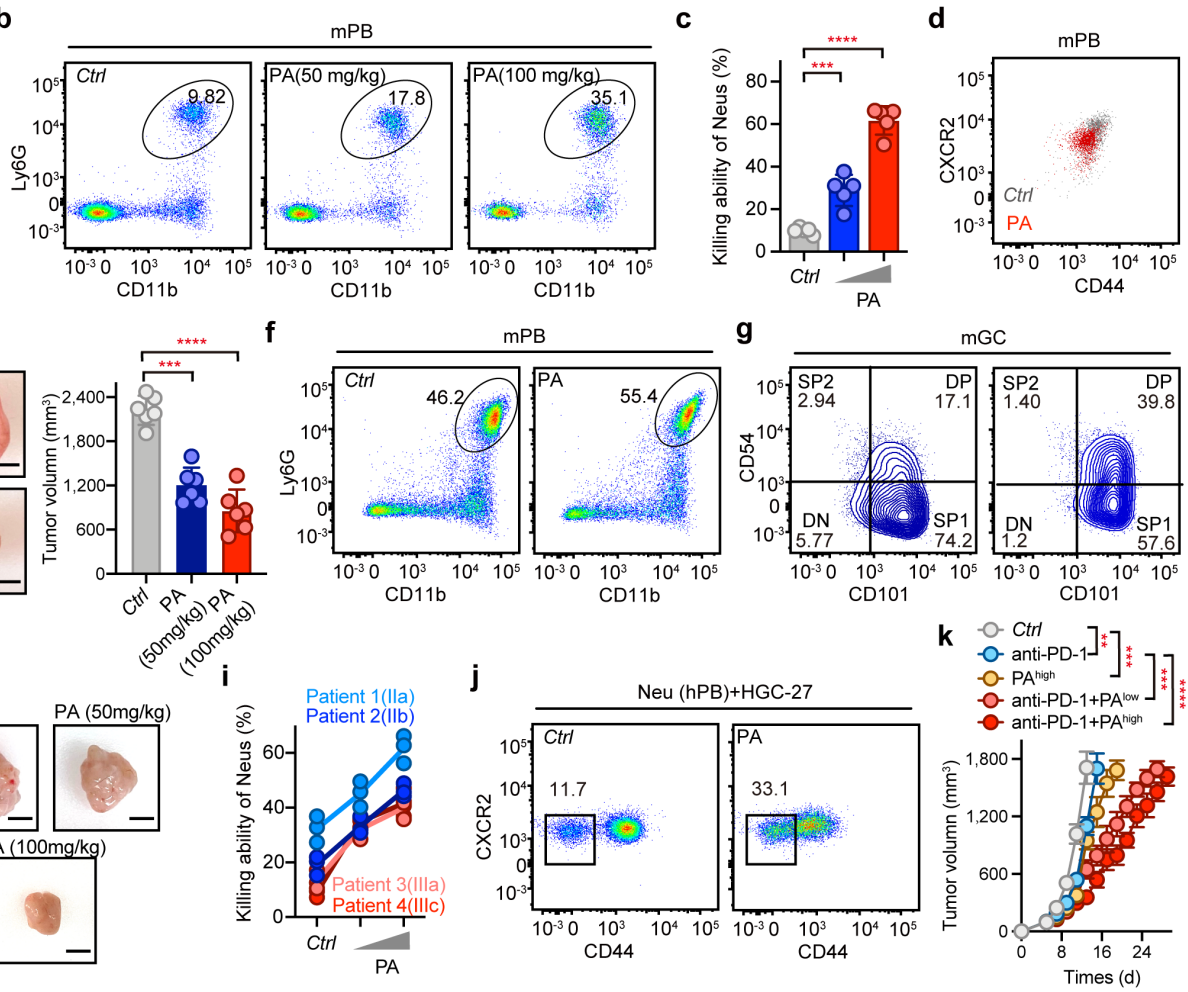

Figure 7 Activation of YAP/TAZ in Neu Reverses GC Progression. (a) Experimental workflow of in vitro neutrophil tumor-killing assay after incubation with YAP/TAZ agonist phosphatidic acid (PA). (b) CD11b ${ }^{+} \mathrm{Ly} 6 \mathrm{G}^{+} \mathrm{Neu}$ in the murine peripheral blood (PB) after treatment with PA for 3 days. (c) In vitro tumor-killing ability of murine $\mathrm{Ly}_{6 \mathrm{G}}{ }^{+} \mathrm{Neu}$ after incubation with PA. (left). Bar graph showing the percentage of Neu tumor-killing activity $(n=5)$. (d) Flow cytometric plots of $\mathrm{CD}^{-}{ }^{-} \mathrm{CXCR2}{ }^{-}$Neu from PA-treated murine peripheral blood. (e) In vivo tumor formation in nude mice after treatment with PA $(n=6)$. Workflow of MFC-induced tumor formation assay (left). (f) CD11 b ${ }^{+} \mathrm{Ly} 6 \mathrm{G}^{+} \mathrm{Neu}$ in PA-treated murine peripheral bloods. (g) tsNeu subpopulations in PA-treated murine tumor tissues. (h) Transfer PA-treated Neu to tumor-bearing recipients $(n=6)$. (i) In vitro killing assay of PA-treated human Neu from GC patients $(n=4)$. (J) Flow cytometric plots of CD44 CXCR2 Neu in PA-treated peripheral blood from GC patient after challenged with HGC-27. (k) A combined therapy (PA and anti-PD-1) in treating melanoma $(n=10)$. PA, 4 times; anti-PD-1, 4 times. See also Supplementary Figure 7. 


\section{Supplementary Files}

This is a list of supplementary files associated with this preprint. Click to download.

- Suppl.pdf 